\title{
基于环糊精模板法的有机合成
}

\author{
张华承刘召娜辛飞飞 郝爱友* \\ (山东大学化学与化工学院 济南 250100)
}

\begin{abstract}
摘要 利用 “模板” 理念综述了基于环糊精模板法的有机合成反应，并将基于环糊精模板法的有机合成反应分为两大 类. 第一类是基于环糊精 “静态模板法” 的有机合成, 主要是指以环糊精刚性结构主、次面和空腔为模板进行的有机 反应. 其中，基于环糊精主次面的有机反应主要是体现了模板效应对空间结构的限定. 而基于环糊精空腔的有机反应， 重点是介绍那些利用环糊精空腔的手性和空间限定性等特点进行化学选择、区域选择和立体选择的反应，这体现了模 板效应中的 “信息” 传递性. 第二类有机反应则是基于 “动态模板法” ，该模板自身是可调控的. 其中，既可以具有可 变空间构象的桥连环糊精二聚体为模板, 也可以环糊精衍生物以及环糊精主客体包合物的自组装体系为模板.
\end{abstract}

关键词 环糊精; 模板法; 有机合成; 应用; 催化

\section{Organic Synthesis Based on Cyclodextrins Template Method}

\author{
Zhang, Huacheng Liu, Zhaona $\quad$ Xin, Feifei Hao, Aiyou* \\ (School of Chemistry \& Chemical Engineering, Shandong University, Jinan 250100)
}

\begin{abstract}
The organic synthesis based on cyclodextrin template method is reviewed initially by using the "template" concept. These organic synthesis reactions are divided into two main types: one type is based on cyclodextrin "static" template which includes the primary, secondary faces and the cavity. The reactions based on the primary and secondary faces mainly show that the template can control the stereo-structure. While the reactions based on the cavity are mainly using the size and chiral properties of the cavity to do the chemical, regio- and stereo-selectivity, showing that the template can carry out information transfer. The other type is based on cyclodextrin "dynamic" template including the bridge-linked cyclodextrin dimmers with variable conformations and the assemblies prepared by the cyclodextrin derivatives and inclusion complexes. These dynamic templates themselves can be adjusted.
\end{abstract}

Keywords cyclodextrin; template method; organic synthesis; application; catalysis

具有轴对称性的、截雉形环状低聚糖分子环糊精 (cyclodextrins, CDs, 图 1) 是超分子化学中一类重要的主 体化合物 ${ }^{[1]}$. 通常根据环糊精结构所含有 $\alpha-1,4-$ 吡喃葡 萄糖单元数量的不同 $(6 \sim 8)$, 将其相应的命名为 $\alpha$-, $\beta$-和 $\gamma$-环糊精. 环糊精分子的空腔是弱极性的(疏水性), 而 外表面则是强极性的(亲水性). 在环糊精的主面或者次 面(primary face and secondary face, 图 1)引入修饰基团, 可以使其物化性质得到改良, 成为具有某种特性的功能 分子. 如果同时在环糊精的同一面引入两个或两个以上 官能团, 为了方便命名, 可以对该面的吡喃葡萄糖结构 单元进行字母编号 ${ }^{[2]}$. 此外, 环糊精结构中的疏水空腔 可以与种类繁多的化合物形成包合物. 同时, 这种富电 性的空腔结构还可以改变所包合的客体分子的电性环
境 ${ }^{[3]}$. 因此，环糊精常被应用到有机合成反应中，发挥 “人工模拟酶” 的作用 ${ }^{[4 ~ 7]}$, 使得反应能够高效、选择性、 温和、环境友好地发生. 因此, 环糊精催化的有机反 应 $^{[8,9]}$ 可以与绿色合成化学 ${ }^{[10 ~ 12]}$ 、超分子催化 ${ }^{[3]}$ 以及微反 应器 ${ }^{[13]}$ 等概念结合, 是近年来有机催化领域研究的热 点之一.

但是，传统思维模式中，基于环糊精的有机反应， 往往只是指在水相中 ${ }^{[14]}$, 客体分子在疏水作用力的驱 动下, 以环糊精空腔为 “相转移载体” 完成的催化反 应 ${ }^{[15 ~ 18]}$. 这种思维模式只是注意到了环糊精个体刚性 结构中 “空腔” 的疏水性 ${ }^{[19]}$ 在有机反应中的作用和应用, 而忽略了环糊精刚性的主、次面，刚性空腔的尺寸和它 对反应的 “选择性” 控制, 以及环糊精可能的自组装形

*E-mail: haoay@sdu.edu.cn

Received April 22, 2011; revised July 1, 2011; accepted July 14, 2011. 

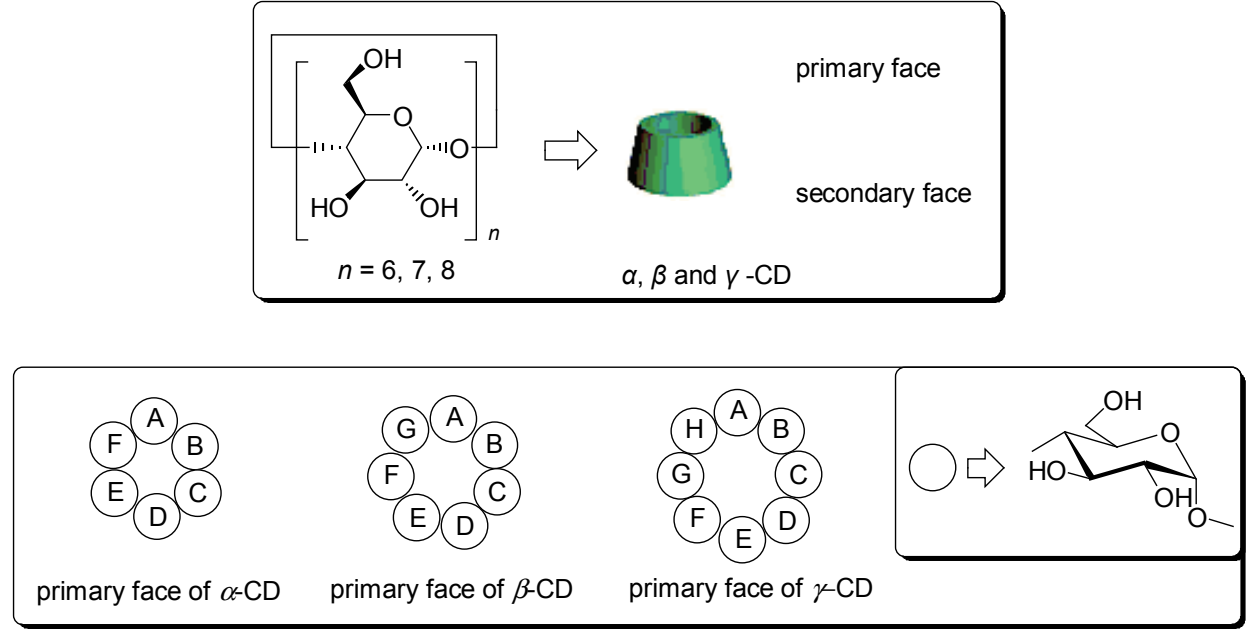

图 1 环糊精的结构

Figure 1 Structure of cyclodextrin

态在有机合成中的作用(图 2). 在系统查阅和总结相关 文献的基础上，本文采用了 “环糊精模板法” 的理念, 基 于环糊精模板法的有机合成不同于以往的单纯的环糊 精催化反应, 既充分考虑到环糊精的结构以及其可能的 自组装形态在有机合成中的作用, 对近年来基于环糊精 模板法的有机合成作了较为全面的介绍和讨论(图 2), 以方便广大科研工作者在相关领域进行更加深入的开 发和应用。
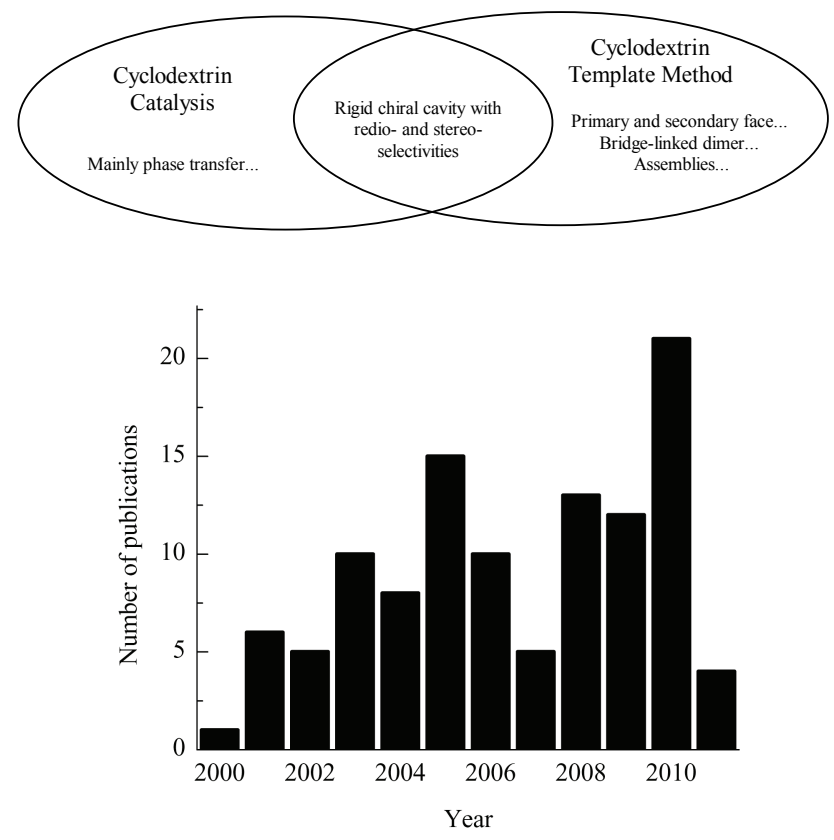

图 2 环糊精模板法与环糊精催化反应的关系及近十年来发 展趋势

Figure 2 Cyclodextrin template method relationship with cyclodextrin catalysis and its trends in recent ten years

就像传统的中国月饼的制作工艺一样，可以通过
“模板” 限制产物的结构, 环糊精就是一个理想的 “模 板” ，即反应产物的结构和大小要与作为反应模板的特 定环糊精的 “结构尺寸” 相吻合. 当然，这里的 “模板” 效应不仅仅是空间结构或者构象上的限定，而且还有 “信息”上的传递. 如同 DNA 复制过程中的 “模板” 效

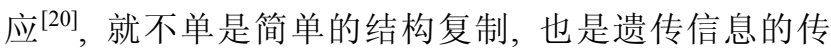
递. 根据这一理念, 我们把基于环糊精模板法的有机反 应分为两大类(图 3): 第一类有机反应是以环糊精刚性 结构为模板进行的. 这里的刚性结构主要指主、次面和 手性空腔. 由于主、次面和空腔结构稳定, 故可以称为 “静态模板法” . 其中, 基于环糊精主、次面的有机反应 主要是体现了空间结构上的限定. 本文中涉及的基于环 糊精空腔的有机反应, 重点是介绍那些利用环糊精空腔 的手性和空间限定性等特点进行化学选择、区域选择和 立体选择的反应. 需要指出的是, 通过环糊精空腔实现 的结构 “信息传递”，主要是指反应产物所具有的区域 和立体选择性. 第二类有机反应则是基于 “动态模板法” 的. 其中, 既可以桥连环糊精二聚体的空间构象为模板, 也可以环糊精衍生物以及环糊精主客体包合物的自组 装体系为模板. 动态模板法的研究目前只是 “初露端 倪”，但却是环糊精静态模板法在有机合成中的新发展 和延伸, 非常符合超分子自组装化学的特色和发展方向 (图 3).

\section{1 静态模板法}

\section{1 以环糊精的主、次面为模板}

环糊精的主、次面具有很好的刚性闭合环形结构, 这就为合成环状分子或者固定链长的低聚合分子提供 了一个天然模板. 同时, 环糊精还可以用来模拟自然界 的酯降解酶. 如果利用这些性质, 通过酯键在环糊精的 


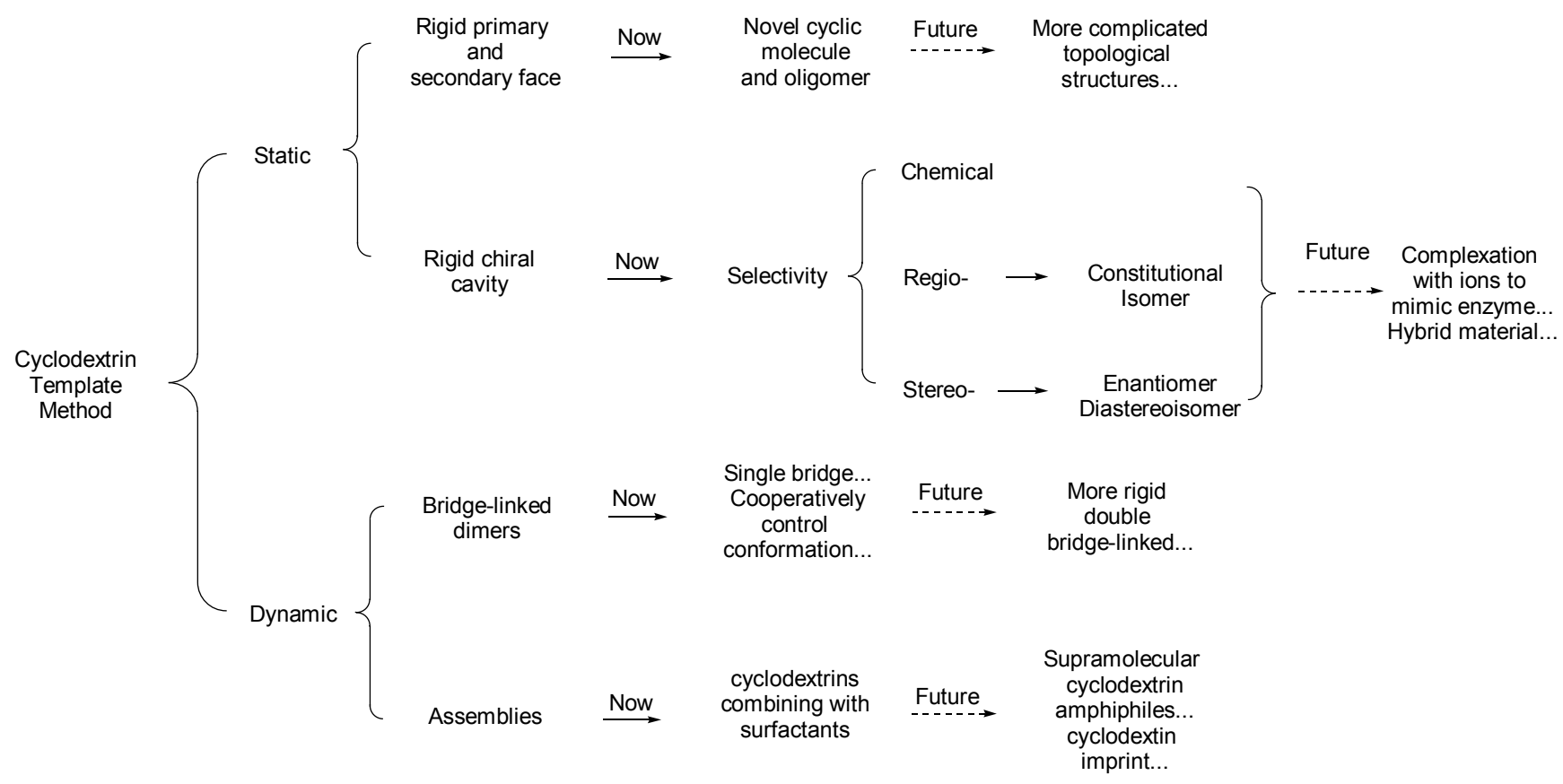

图 3 环糊精模板法的 “谱系”

Figure 3 Family tree of cyclodextrin template method

主、次面进行 “环形” 修饰, 那么很容易得到所需要的 环状或者固定链长的低聚合分子. 例如, 主、次面分别 被乙酰基化和甲基丙烯酸䣶酯化的 $\beta$-环糊精衍生物被 首次用作模板 ${ }^{[21]}$, 进行了基于次面刚性结构的可控自 由基聚合反应(radical polymerization). 这里所采用的可 控自由基聚合反应的引发剂是 $p$-二甲苯基- $N, N$-二甲基 二硫代氨基甲酸酯 ( $p$-xylyl- $N, N$-dimethyldithiocarbamate, $\mathrm{XDC}$ )和 $\alpha$-溴代- $p$-二甲苯基- $N, N$-二甲基二硫代氨基甲 酸酯 ( $\alpha$-bromo- $p$-xylyl- $N, N$-dimethyldithiocarbamate, BX$\mathrm{DC}$ ). 所得到的次面外接 “封闭” 环状低聚分子的 $\beta$-环 糊精衍生物可以进一步水解得到环状低聚甲基丙烯酸.

原子转移自由基聚合反应(atom transfer radical polymerization, ATRP)结合了自由基聚合以及活性聚合反 应的优点, 并且更加简洁、高效、可控，故被引入到基 于环糊精刚性主次面为模板的反应中来. 以 1,3 -二溴丁 烷为引发剂、溴化铜为催化剂、五甲基二乙烯三胺为配 体，通过 ATRP 反应 ${ }^{[22,23]}$ 使修饰在 $\beta$-环糊精次面的甲基 丙烯酸基发生聚合反应，得到次面外接有 “不封闭” 环 状低聚分子的新型 $\beta$-环糊精衍生物. 温度和溶剂对该反 应的影响也做了具体研究. 以二吡啶被用作配 体 ${ }^{[23,24]}$, 还得到了主、次面均有修饰的化合物 1 (Scheme 1). 然后, 在碱性环境下, 外接的低聚甲基丙烯酸酯发 生酯解反应, 脱离 $\beta$-环糊精模板, 得到具有固定链长的 低聚甲基丙烯酸(Scheme 1). 含有芳香环及长疏水链结 构的客体分子对环糊精模板法合成低聚甲基丙烯酸的 聚合度也有影响 ${ }^{[25]}$. $\alpha$-, $\beta$-环糊精的主面也可以单独被用
作模板 ${ }^{[31,34,35]}$, 以同样的方法得到了不同链长的低聚甲 基丙烯酸.
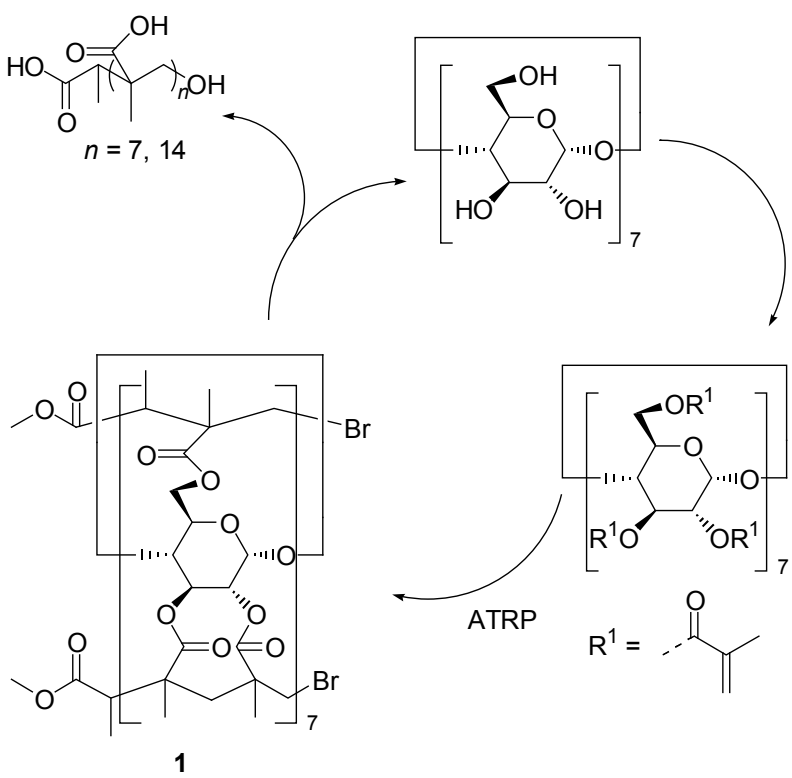

Scheme 1

1,3-二胺基丙烷或者 1,4-二胺基丁烷可用来将上文 中提到的次面外接有 “不封闭” 环状低聚分子的 $\beta$-环糊 精衍生物 $\mathbf{2}$ 的 “缺口” 封闭 ${ }^{[23,26]}$, 得到了次面外接有 “封 闭” 环状低聚物的新型 $\beta$-环糊精衍生物 3 (Scheme 2).

另外, 如果按照 ATRP 的反应条件 ${ }^{[27]}$, 将次面 “不 封闭” 环状低聚分子的 $\beta$-环糊精衍生物 2 用来继续进行 反应 ${ }^{[23]}$, 结果发现 ATRP 反应还可以进行, 也可得到次 

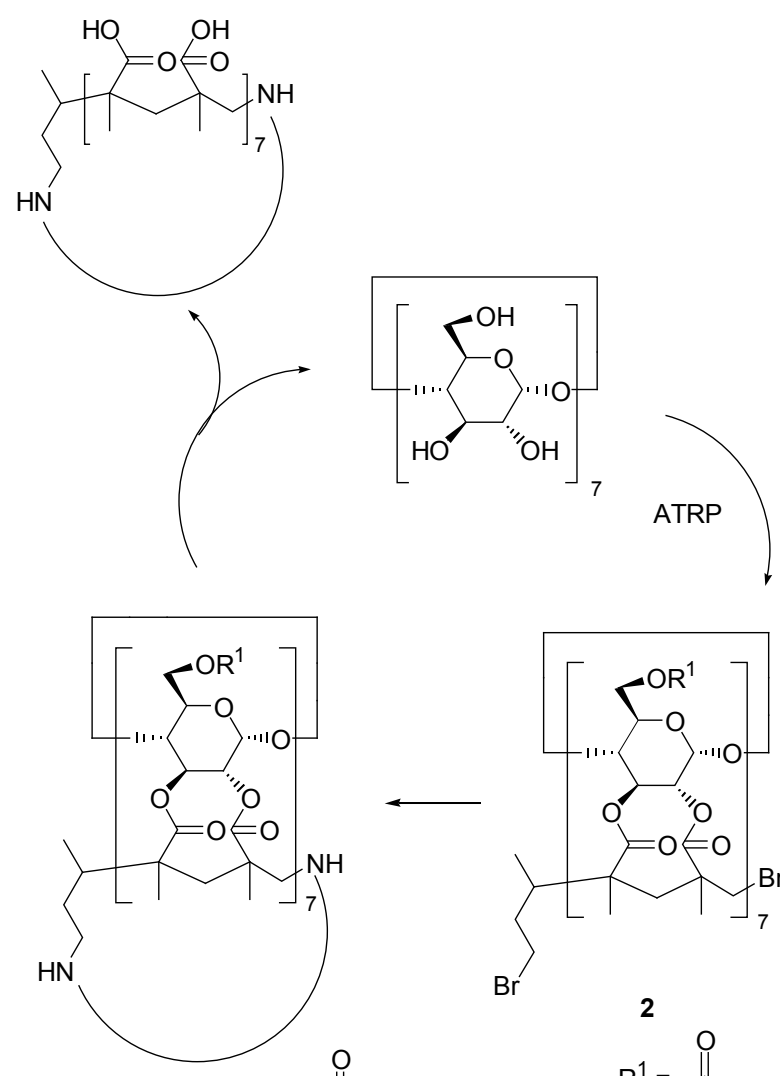

$\mathrm{R}^{1}=$,

\section{Scheme 2}

面外接有 “封闭” 环状低聚分子的 $\beta$-环糊精衍生物.

采用类似的方法，主、次面都外接有 “封闭” 环状 低聚分子的 $\beta$-环糊精衍生物 4 也被合成出来，只是主、 次面的外接环状低聚分子的大小不同(Scheme 3). 在完 成酯解反应后, 得到了系列具有不同尺寸的环状低聚甲 基丙烯酸(Scheme 3). 与其他合成环状低聚甲基丙烯酸 的方法相比, ATRP 参与的环糊精模板法更高效、更具有 可控制性. 而且, 所得到的新型环状低聚甲基丙烯酸具 有很好的对甲基蓝的客体识别功能, 是一种新型的主体 化合物 ${ }^{[28]}$.

\section{2 以环糊精的空腔为模板}

以环糊精空腔为载体进行的有机合成反应类型丰 富，几乎覆盖了所有有机合成反应的主要种类. 这些反 应既可以在固相条件下进行, 也可以在液相条件下进 行. 其中, 液相反应又可以分为在极性溶剂或者非极性 溶剂中进行. 无论反应条件如何, 所利用的环糊精空腔 特性主要是该空腔可以与客体分子形成稳定的包合物. 而包合作用的强弱又与客体分子的大小、形状及分子的 匹配程度等有关. 当使用 “模板法” 的理念来考察那些 利用环糊精空腔进行的有机反应时, 空腔对反应底物体 积大小的限制(化学选择性), 以及反应底物与产物之间 的 “信息” 传递(区域和立体选择性)就变得非常重要了.
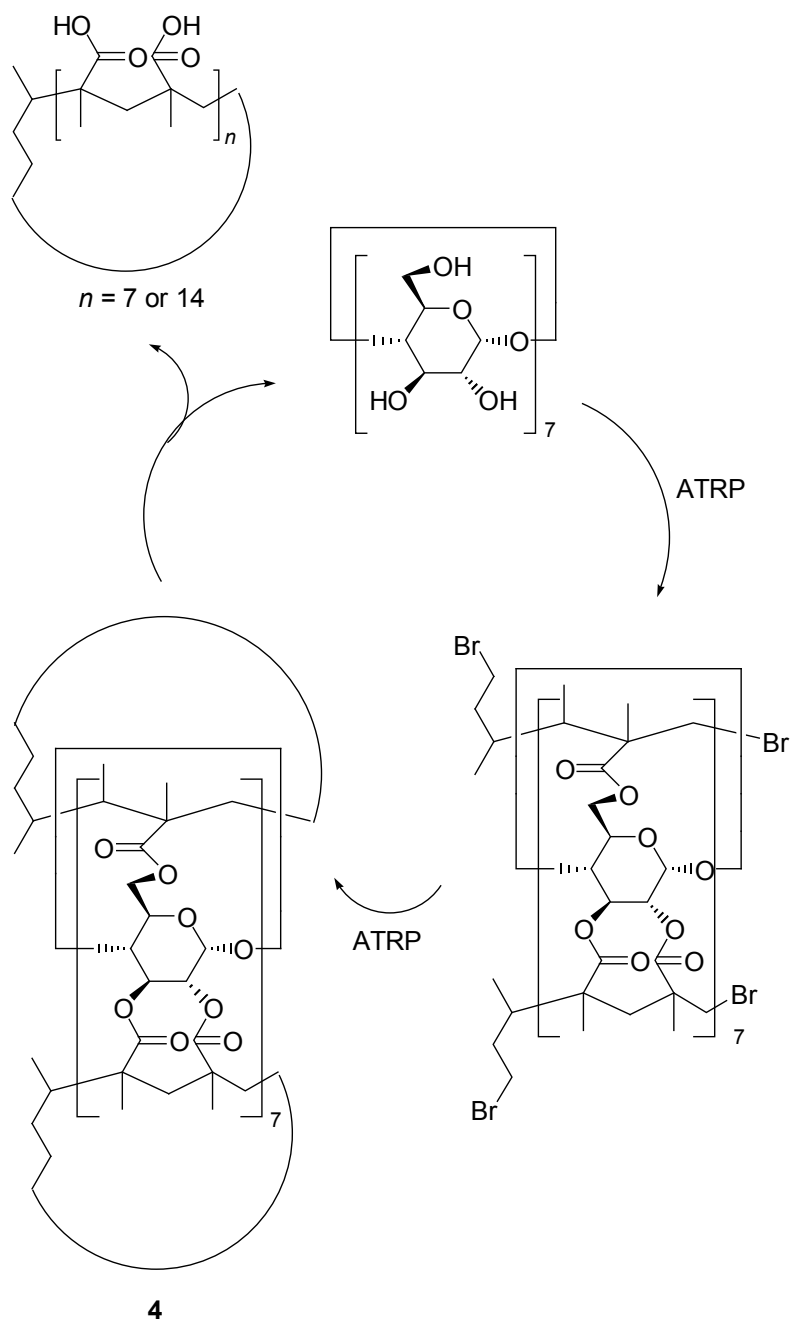

Scheme 3

\section{2 .1 化学选择性}

环糊精为模板的有机反应的化学选择性主要体现 在对底物体积和形状等因素的控制上，即只有那些与环 糊精空腔尺寸相吻合的反应底物才更容易借助空腔实 现反应或者提高反应效率，这方面的实例非常多，几乎 贯穿环糊精相关催化反应的始终，而且基本原理类似， 故以下只举几个氧化反应的例子进行简要地分析说明.

在有机氧化反应中, 作为一种高效的催化剂, $\mathrm{FeBr}_{3}$ 环糊精复合体系可以参与氧化硫醇或硫醚生成砜 类化合物的反应 ${ }^{[29]}$. 反应中, $\mathrm{FeBr}_{3}$ /环糊精复合体系可 以根据底物分子的尺寸等条件, 选择性地与底物分子形 成包合物, 体现了对底物分子的化学选择性 ${ }^{[30]}$, 起到了 反应模板的作用. 水相中以次氯酸钠作为氧化剂氧化醇 成醛的反应中, 起模板催化作用的 $\beta$-环糊精 ${ }^{[31]}$ 在反应底 物的选择上, 空间因素比电性因素起的作用更大, 含有 芳香基的醇类氧化产率往往高于单纯的脂肪醇. 2-碘酰 苯甲酸(IBX) 是一种典型的高价碘试剂，常被用于有机 氧化反应中 ${ }^{[32]}$. $\beta$-环糊精存在时 ${ }^{[33]}$, 氧杂环丙烷、呋丙啶 
等与 2-碘酰基苯甲酸(2-iodoxybenzoic acid, IBX)作用, 可高选择性、高收率地氧化生成 $\alpha$-差基酮和 $\alpha$-氨基酮. 又如, 醛、酮肜与 2 -碘酰苯甲酸作用生成相应醛酮的反 应中 ${ }^{[34]}$, 起模板催化作用的 $\beta$-环糊精有选择性地与底物 分子进行复合. 底物分子的尺寸与 $\beta$-环糊精空腔匹配得 越好, 模板效应越明显, 催化效果越好.

除此之外, 以环糊精空腔为模板对反应底物尺寸表 现出的类似的化学选择性还可以在其它种类的氧化反 应 $^{[35 \sim 42]}$ 、还原反应 ${ }^{[43]}$ 、水解反应 ${ }^{[44,45]}$ 、加成反应 ${ }^{[46]}$ 、取

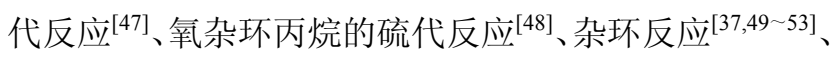
Tsuji-Trost 反应 ${ }^{[54]} 、$ Suzuki 反应 ${ }^{[5]}$ 和电环化反应 ${ }^{[56]}$ 等中 体现. 需要特别指出的是, 具有不同大小空腔的环糊精 分子 ( $\alpha-, \beta$-和 $\gamma$-环糊精) 对相同尺寸的反应底物选择性也 不相同, 模板效应也非常明显. 例如, Tsuji-Trost 脱羧反

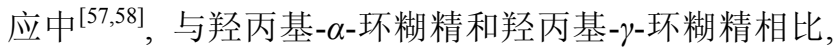
起模板催化作用的羟丙基- $\beta$-环糊精可以与反应底物形 成更稳定的包合物, 因此, 对反应的选择性和催化效率 更高.

\subsection{2 “信息”传递一区域选择性}

环糊精空腔参与的有机反应中 “信息” 传递主要体 现在空腔对反应的区域和立体选择性. 环糊精模板的这 种选择性主要体现在空腔对反应产物尺寸的限制. 比 如, 区域选择性就是通过底物与环糊精空腔形成包合 物, 屏蔽掉底物中的 “干扰性” 反应位点, 使反应集中 在空间位阻较小的位置发生，限制了所得产物的尺寸， 从而实现提高反应选择性的目的.

例如, 卤代反应中, 以 $\alpha$-环糊精参与的茴香醚的选 择性氯化 ${ }^{[59]}$ 为例. $\alpha$-环糊精包合茴香醚, 起到了模板作 用, 使茴香醚中芳环的邻位和间位受到屏蔽, 结果使氯 化反应更易在芳环对位进行, 达到了区域选择的目的. 类似的，在取代苯酚(3-硝基苯酚、2-氯代苯酚、3-氯代 苯酚以及 4 -氯代苯酚)的溴代反应中 ${ }^{[60]}, \beta$-环糊精也发挥 了模板作用, 使单溴代更加容易进行, 多溴代产物减少, 溴代反应主要发生在 3-硝基苯酚中硝基的对位、2-氯代 苯酚中羟基对位、3-氯代苯酚的羟基对位以及 4-氯代苯 酚的羟基邻位. 同样, $\beta$-环糊精参与的间氟苯酚的选择 性碘化反应中 ${ }^{[61]}$, 反应主要在羟基的对位发生, 生成 3氟-4-磑苯酚. 同样是以芳香族化合物为底物的反应, 联 苯 “一锅法” 合成 4,4'-联苯二甲酸 ${ }^{[62,63}$ 和菜 “一锅法” 合成 2,6 -萗二甲酸 ${ }^{[64]}$ 的反应中, $\beta$-环糊精的存在也起到 了模板作用, 可以对羧酸产物的取代位置进行区域选 择.

在烯烃与一氧化碳的亲电加成实现氢甲酰化的反 应中, 天然和修饰的环糊精可以作为模板 ${ }^{[65 ~ 67]}$ 调节两 种加成产物的比例, 其中环糊精空腔与产物的尺寸的匹
配程度对区域选择的结果影响重大. 例如, 正癸烯与一 氧化碳加成的反应中(Eq. 1) ${ }^{[68]}$, 甲基化 $\beta$-环糊精存在 下，反应的转化率可以达到 $62 \% \sim 97 \%$, 该值与环糊精 衍生物的取代度有关, 取代甲基数目越多, 转化率越高; 而 $e e$ 值则始终维持在 $96 \%$, 线形产物(linear)与枝形产物 (branched)的物质的量的比达到 1.8. 水相中二甲基 $\beta$-环 糊精作为金属催化剂的二层(second sphere)配体参与的 氢甲酰化反应中 ${ }^{[69]}$, 产物的转化率最高达到 $76 \%, e e$ 值 达到 $95 \%$, 而线形与枝形产物的物质的量的比值达到了 4.3.

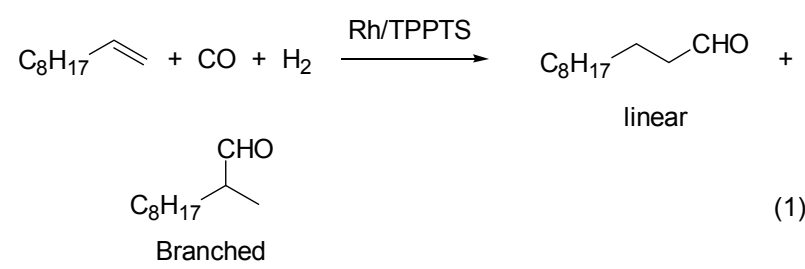

环糊精空腔模板对有机合成的区域选择性控制在 成环和开环反应中表现得更为明显. 例如，甲基苯醌和 2-甲基-1,3-丁二烯的 Diels-Alder 反应 ${ }^{[70]}$, 加入 $\beta$-环糊精 可区域选择性生成间位化合物. 再如, 在 $\beta$-环糊精催化 各种苯硫酚对环氧化物的开环反应中 ${ }^{[71]}, \beta$-环糊精与环 氧化物首先通过氢键形成包结物, 然后苯硫酚通过 $\beta$-环 糊精的窄口端进攻开环，可以制备 $\beta$-羟基硫醚类化合 物, 区域选择性良好. 酚钠盐进攻氧杂环丙烷的开环反 应中 ${ }^{[72]}, \beta$-环糊精也可以发挥模板作用，高区域选择性 地生成 $\beta$-差基醚. 以卤化氢和卤化锂为进攻试剂, 在 $\beta$ 环糊精存在下, 氮杂环丙烷可高选择性地开环生成 $\beta$-卤 代胺 ${ }^{[73]}$. 类似的, 在 $\beta$-环糊精参与下 ${ }^{[74]}$, 亲核性的芳香 硫醇和胺与 2,3 -氮杂环丙烷反应生成对应的 $\beta$-氨基硫化 物和邻二胺化合物, 其机理与环氧开环类似. 同样的, 有 $\beta$-环糊精存在的芳基氮杂环丙烷的开环氧化反应 ${ }^{[75]}$ 以 $\mathrm{NBS}$ 为氧化剂在水相中 $50{ }^{\circ} \mathrm{C}$ 发生, 可以高产率地生 成 $\alpha$-氨基酮，区域选择性原理与其它的氮杂环丙烷开环 机理类似.

又如, $\beta$-环糊精存在时 ${ }^{[76]}$, 乙酸-1-菜酯、乙酸-2-䒬 酯、苯甲酸-1-䒬酯和苯甲酸-2-菜酯的光促 Fries 重排反 应可高效地选择性生成邻位产物. 如果使用 $\gamma$-环糊精为 模板 ${ }^{[77]}$ 可以进一步提高邻位取代产物的选择性. 环糊 精模板对 Reimer-Tiemann 反应也有区域选择性催化作 用 ${ }^{[78]}$, 可以使反应选择性地生成对羟基芳醛. 将 $\alpha$-环糊 精与 $\beta$-环糊精作比较发现, 由于后者对苯酚的邻位有屏 蔽作用，故其对位选择性更好. 同时，修饰的 $\beta$-环糊精 由于与对着基苯甲酸的包合作用增强，其区域选择性进 一步增强 ${ }^{[79]}$. 


\subsection{3 “信息”传递一立体选择性}

立体选择性则主要是利用了环糊精手性空腔的模 板效应，进行 “手性” 信息的传递. 其中各种反应条件, 如温度、压力、固液相反应以及溶液相中的极性因素等, 都会对模板效应产生影响. 由于立体选择性反应涵盖了 刚性空腔对底物的化学选择性以及区域选择性的特点, 通过对立体选择性反应的介绍可以较为全面地了解环 糊精刚性空腔模板效应的 “信息传递” 功能以及研究历 程, 故在此处将做详细介绍.

\subsubsection{1 通过电环化反应浅谈其特点及研究历程}

以周环反应中的电环化反应为例, 可以较为全面地 了解环糊精手性空腔的模板效应对有机反应立体选择 性控制的特点及研究历程. 早期的研究认为客体分子被 $\gamma$-环糊精包合后, 仍会移动或者旋转, 导致很难对客体 分子的反应进行立体化学地控制 ${ }^{[80]}$. 因此, 初期利用环 糊精手性空腔作为模板的立体化学反应, 常常将反应底 物共价键连在环糊精的主面上. 这种做法带有很明显的 利用环糊精主面刚性结构模板 “固定” 空间构象的特点. 例如，1-蒽甲酸的二聚反应就是通过 6A,6X-双(蒽-1-羰 基)- $\gamma$-环糊精 $(\mathbf{5})(\mathrm{X}=\mathrm{B} \sim \mathrm{E}$, Scheme 4 )在极性溶液中的 光致催化反应得到的. 由于是液相反应, 还可以通过调 节极性溶剂的组成来改变所生成的 4 种 1-蒽甲酸二聚异 构体 d1-1 d1-4 的比例(Scheme 4).

之后的研究发现, 当 $\gamma$-环糊精存在时, 不将反应底 物共价键连在环糊精的主、次面, 也可以实现光致催化 反应. 也就是说, 完全利用环糊精手性空腔自身的模板 效应，也可以达到控制反应立体选择性的要求. 例如, 以天然 $\gamma$-环糊精手性空腔为模板, 2 -蒽甲酸可以在水相
介质中进行光致二聚反应 ${ }^{[81]}$ ，总收率达到 $46 \%$; 并且, 可以得到手性产物 d2-2 (syn-head-to-tail, syn-HT) 和 d2-3 (anti-head-to-head, anti-HH), 具有很好的立体选择性, 对映体过量(enantiomeric excess, ee)值可达 41\% (Eq. 2), 这在无 $\gamma$-环糊精空腔模板作用时是不能实现的. 而 HT：HH 的选择性也可以达到 $87 ： 13$, 高于无 $\gamma$-环糊精 空腔模板作用时的 78:22. 同时, 发现温度对二聚产物 的立体选择性, 即光学产率有较大影响: 在 $25{ }^{\circ} \mathrm{C}$ 时, ee 值仅为 $32 \%$; 而在 $0{ }^{\circ} \mathrm{C}$ 时, $e e$ 值则为 $41 \%$. 其原因可能 是温度的降低使 2 -蒽甲酸在 $\gamma$-环糊精中的自由度受到 抑制，反而提高了反应体系的立体选择性 ${ }^{[56]}$. 另外，高 压也对提高反应的立体选择性有利, 但是原因还不明 确 ${ }^{[82]}$.
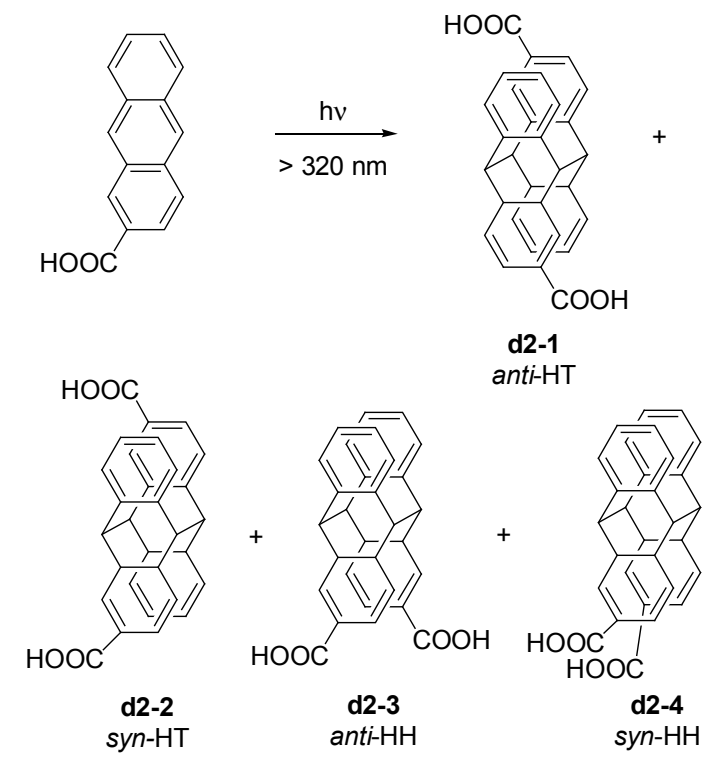

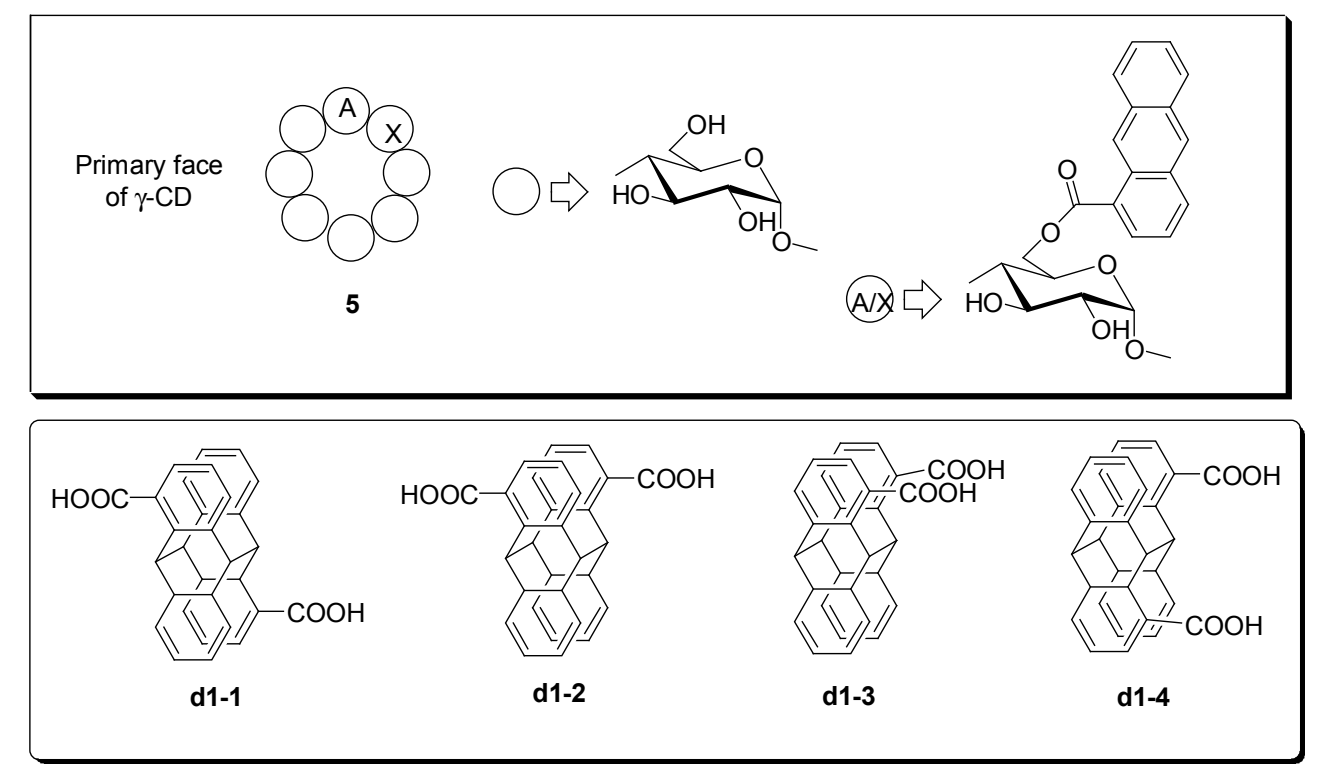

Scheme 4 
离子型环糊精衍生物手性空腔在立体选择性中的 模板效应也得到了进一步研究. 例如, $\gamma$-环糊精主面 A,E-位置修饰有两个阳离子吡啶官能团的新型主体化 合物 6 也可作为模板, 控制 2-蒽甲酸的光致二聚反应 (Scheme 5) ${ }^{[83,84]}$. 相对于 $\gamma$-环糊精和修饰有一个吡啶基 团的 $\gamma$-环糊精, 这种新型的主体化合物在立体选择性上 与天然 $\gamma$-环糊精类似, 但却使 2-蒽甲酸二聚体异构体 d2-1 和 d2-3 的区域选择性大幅增强(Eq. 2). 延续这一思 路, 在天然 $\gamma$-环糊精的主面单修饰一条含有两个阴离子 识别位点的柔性链, 可以得到 $6^{\mathrm{A}}$-[2-(二甲胺基) 乙 胺]- $6^{\mathrm{A}}$-脱氧- $\gamma$-环糊精(7) (Scheme 5) ${ }^{[56]}$. 研究发现, 利用 这一主体化合物为模板, 一方面可以大体保持与天然 $\gamma$ 环糊精类似的立体选择性; 另一方面, 可以提高二聚体 异构体 d2-3 和 d2-4 的产率. 究其原因, 主要是因为柔性 链上的阴离子识别位点起到了 “模板” 效应, 可以通过 离子键作用，“固定”反应底物的空间构象.

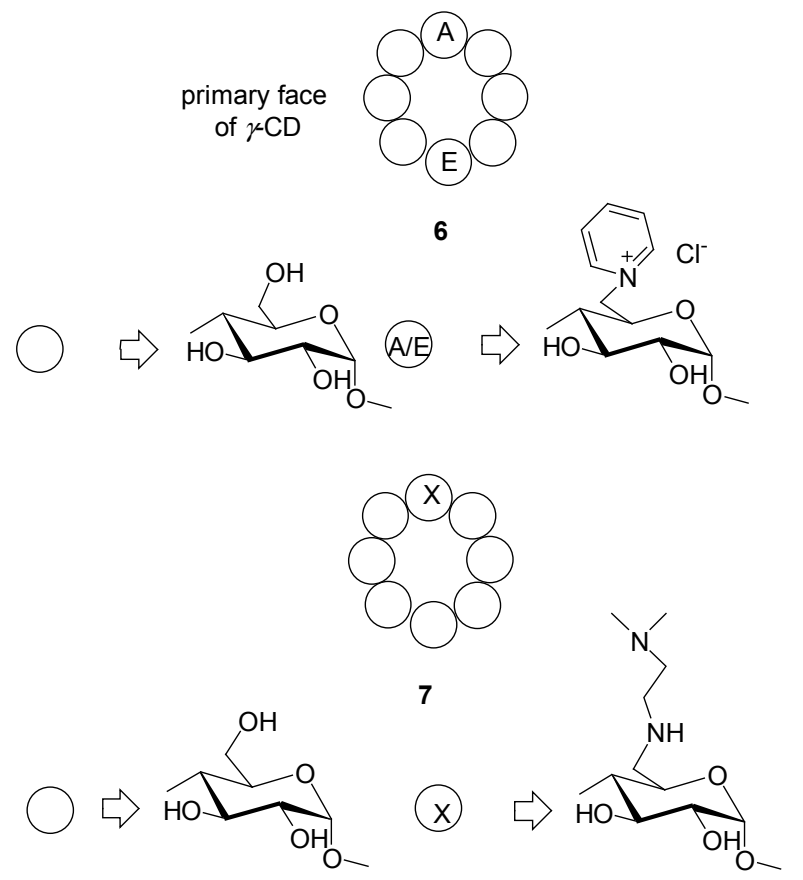

\section{Scheme 5}

如果使含有二胺基官能团的 $\gamma$-环糊精衍生物与二 价金属离子, 如铜(II)离子等配位, 那么这种被金属配 位的侧链将会增强 2-蒽甲酸与环糊精手性空腔的包合 作用，同时还可以控制 2-蒽甲酸在空腔内的空间构象， 增强其光致二聚反应的立体选择性 ${ }^{[85]}$. 例如，在－50 ${ }^{\circ} \mathrm{C}$, 水和甲醇的混合体系中, 只需 0.1 equiv. 的 $6^{\mathrm{A}}$-[2-(二 甲胺基)乙胺]- $6^{\mathrm{A}}$-脱氧- $\gamma$-环糊精(7) (Scheme 5)与铜(II)离 子的金属配位的主体化合物, 就可以使二聚异构体 d2-3 (Eq. 2)的产率达到 51\% 52\%, ee 值达到 64\% 70\%. 此 外, 环糊精衍生物 $6^{\mathrm{A}}, 6^{\mathrm{X}}$-二胺基- $6^{\mathrm{A}}, 6^{\mathrm{X}}$-脱氧- $\gamma$-环糊精

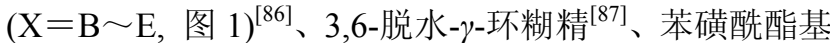
主面修饰的 $\gamma$-环糊精 ${ }^{[88,89]} 、 6^{\mathrm{A}}, 6^{\mathrm{E}}$-间甲苯酚二苯并咪唑 “帽型” $\gamma$-环糊精 ${ }^{[90] 、 6-(~} \gamma$-氨基烷基胺基)- $\gamma$-环糊精 ${ }^{[91]}$ 以 及一系列次面修饰的 $\gamma$-环糊精衍生物 ${ }^{[82]}$ 等也有类似的 模板效应，可以在 2-蒽甲酸的光致二聚反应中发挥立体 选择的作用.

如果将 2-蒽甲酸修饰到 $\alpha$-环糊精的主面, 以这个偶 联化合物 8 作为反应底物, 仍以 $\gamma$-环糊精的手性空腔为 模板, 对 2-蒽甲酸亚结构进行水溶液中的光致二聚催化 反应。结果得到了以 $\alpha$-环糊精封端的新型轮烷中间产物 9, 10 ${ }^{[92]}$, 总收率可以达到 95\% (Scheme 6). 这也提供了 一种非常高效的合成 $\gamma$-环糊精轮烷的方法. $\alpha$-环糊精亚 结构在这个反应中的作用是增强了反应底物的水溶性, 提高了反应效率. 随后, 轮烷 9,10 在 $2 \mathrm{~mol} \cdot \mathrm{L}^{-1}$ 的氢氧 化钾水溶液中水解, 最终得到了 2-蒽甲酸的二聚体产物 (Eq. 2). 其中, $\mathbf{d 2 - 2}$ 的 $e e$ 值在 $25{ }^{\circ} \mathrm{C}$ 就可以达到 $81 \%$, 在 $-20{ }^{\circ} \mathrm{C}$ 就更是达到了 $91 \%$; 而 HT : HH 的选择性也可 以达到 $98: 2$, 远高于上文提到的无修饰的 2-蒽甲酸光 致二聚时的 $87: 13$ (Eq. 2).
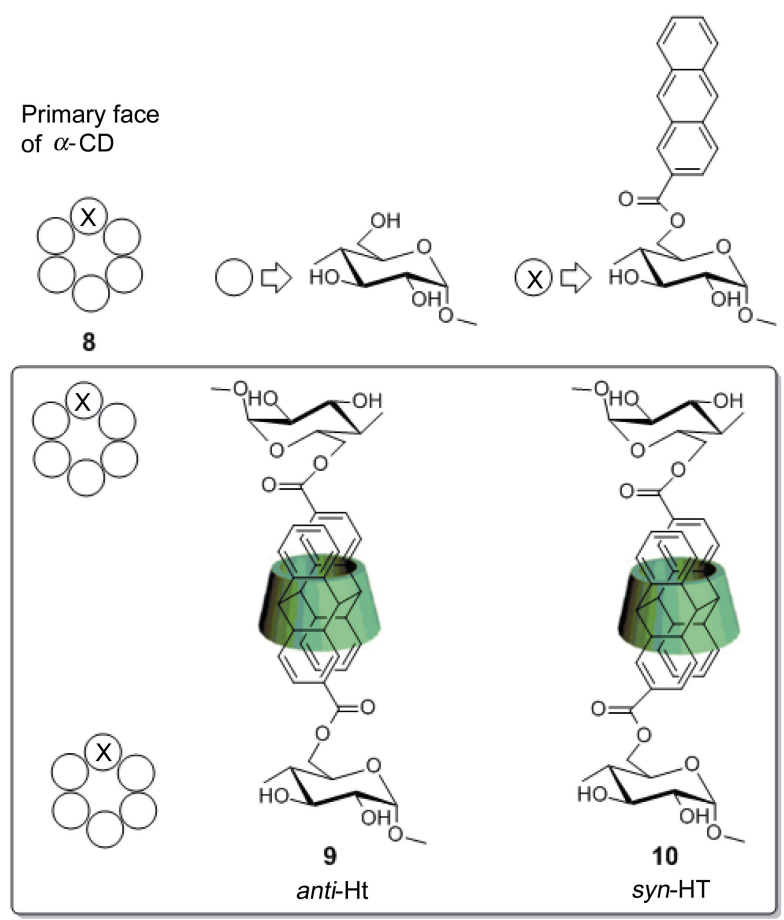

\section{Scheme 6}

值得特别注意的是，两个手性源可以同时对 2-葱甲 酸的光致二聚反应的立体选择性产生影响. 例如, 将 2葱甲酸共价键连到直链淀粉上得到取代度为 $51 \%$ 的 6-O-(2-蒽甲酸)支链淀粉(Ac-AM, Scheme 7) ${ }^{[93]}$. 没有 $\gamma$ 环糊精存在时，其在二甲基亚砜的水溶液中的光致二聚 反应主要得到 $\mathrm{HH}$ 型二聚体(Eq. 2), 且 d2-2 和 d2-3 的 
$e e$ 值仅分别为 $12 \% \sim 15 \%$ 和 $1 \% \sim 2 \%$. 加入 $\gamma$-环糊精后, 反应选择性地生成 HT 型二聚体, 产率由原来的 $29 \%$ 提 高到 76\%; 且 $\mathbf{d 2} 2-2$ 的 $e e$ 值提升到 37\%, 而且 $\mathbf{d 2} 2-3$ 的 e.e. 值可以通过调节激发温度来控制. 在这里支链淀粉结构 起到了手性构架的作用，与环糊精的手性空腔共同作用 于光致二聚反应中产物的立体选择. 类似的光致环化反 应, 比如, 3-甲氧基-2-菜甲酸甲酯的光致二聚环化反 应 ${ }^{[94]}$ 也可以被 $\gamma$-环糊精手性空腔控制(Scheme 8). 水溶 液中的二聚体产物的产率为 $85 \%, e e$ 值为 $48 \%$; 而固相 中产率为 $78 \%, e e$ 值仅为 $34 \%$. 据分析, 在水相反应中, 环糊精手性空腔的疏水效应对于控制底物分子在空腔 中的构象起到了非常重要的控制作用. 故此反应中, 水 相反应的立体选择性高于固相反应.

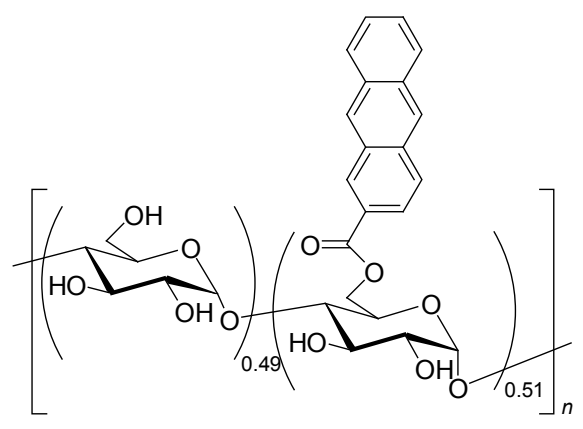

AC-AM

Scheme 7

2
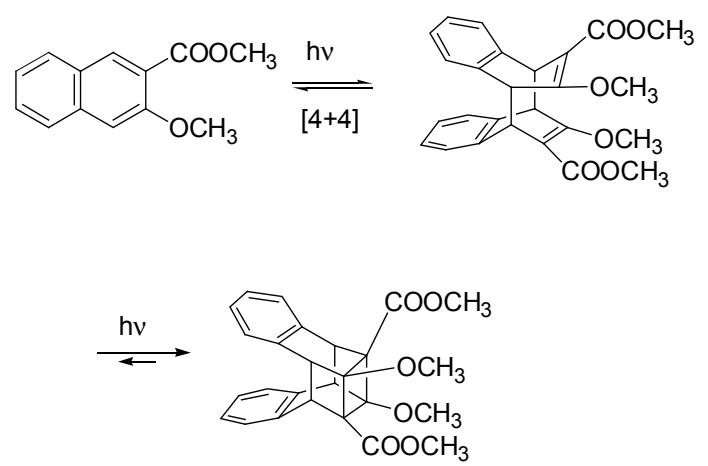

Scheme 8

对映差向光异构反应同样可以利用环糊精手性空 腔的模板效应提高其立体选择性. 例如, 在水和甲醇的 混合溶液中, 具有前手性的 $(Z)$-环辛烯(1Z)向手性 $(E)$-异 构体(1E)的对映差向光异构反应(Scheme 9), 可以通过 加入含有系列光敏化剂(sensitizer)苯甲酸基团的 $\beta$-环糊 精衍生物来控制 $(E)$-环辛烯的光学产率 ${ }^{[95,96]}$, 最高可以 达到 $46 \%{ }^{[97,98]}$. 在这一过程中, 溶剂的极性、温度、主 客体的包合强度、环糊精空腔的占有率以及苯甲酸修饰 基团的体积和位置等都会对立体选择的结果产生影响. 另外, 在 $(Z, Z)-1,3$-环辛二烯(1ZZ) 向 $(E, Z)$-1,3-环辛二烯
异构体(1EZ)的转化中，萗基修饰的环糊精空腔同样发 挥了立体选择性控制的作用(Scheme 9) ${ }^{[99]}$. 研究发现, $\beta$ 环糊精衍生物与底物分子的包合强度最大; $\alpha-, \beta$-环糊精 衍生物因具有较小的空腔结构, 其包合物具有低熵值的 特点, 因此对温度的变化不如 $\gamma$-环糊精衍生物敏感. 因 此, 相对于 $\alpha-, \gamma$-环糊精衍生物, 䒬基修饰的 $\beta$-环糊精可 以使该反应达到中等的 $e e$ 值 $4.6 \%$.

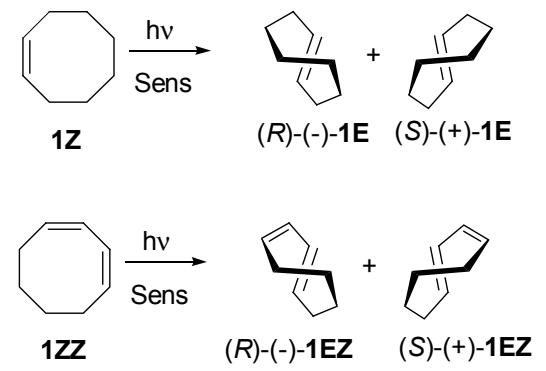

Scheme 9

\subsubsection{2 固相反应与液相反应的影响}

在以环糊精空腔为模板的有机反应中, 采用不同的 反应条件一一固相反应或者液相反应, 会对产物的立体 选择性产生非常重大的影响. 早期的研究认为, 固相条 件更有利于手相空腔发挥模板作用. 例如, 在环糊精存 在下的光促托酚酮(tropolone, 既环庚三烯酚酮)环化反 应中 ${ }^{[100]}$, 固相反应产物的 $e e$ 值可达 $33 \%$, 而采用水相 反应时的产物 $e e$ 值则仅为 $4 \%$. 原文以为, 可能是由于 在固相反应条件下, 底物分子与环糊精可形成包合物, 这为底物分子提供了一个相对更优越的刚性反应模板. 类似的研究进一步表明 ${ }^{[101]}$, 固相反应条件下, 环糊精 分子可以与客体分子形成通道式晶格结构 (channeltype). 因此, 固相反应在此类研究的早期被广泛地作为 环糊精空腔模板立体选择性控制反应产物的基本条件. 比如，在 $\beta$-环糊精存在时 ${ }^{[102]}, N$-烷基吡啶酮的光辐射固 相环化反应产物 2-氮杂二环[2.2.0]-六-5-烯-3-酩(Scheme $10)$ 的 $e e$ 值可达 $60 \%$. 在氧化反应中也有类似的发现.

但是，与之前所述的光致二聚反应类似，这种固相 反应条件优于液相反应条件的影响在氧化反应中表现 的同样不明显, 甚至出现相左的结果. 比如, 利用酮酰 基 $\alpha$-环糊精/过硫酸氢钾(Oxone)体系 ${ }^{[103]}$, 在 $\mathrm{NaHCO}_{3}$ 溶 液中，催化芳香族烯烃的环氧化反应，产生的环氧化合 物以 $R$ 型为主，立体选择性可达 $45 \%$ 以上. 又如，由 Acetobacter aceti 酶不对称氧化 $(R / S)$-2-苯基-1-丙醇合成 (S)-2-苯基-1-丙酸的反应中, 加入 $38 \mathrm{~mol} \%$ 的 $\beta$-环糊精 时 $^{[104]}$, 经过 $6 \mathrm{~h}$ 反应, 产物 $e e$ 值竟高达 $97 \%$, 显著提高 了原有反应的速度、底物的耐受性和产物的对映选择性. 另外, 黄素修饰的 $\beta$-环糊精衍生物 11, 12 可以在以过氧 化氢为氧化剂的、对甲基苯基硫醚的水相亚砜化反应中, 

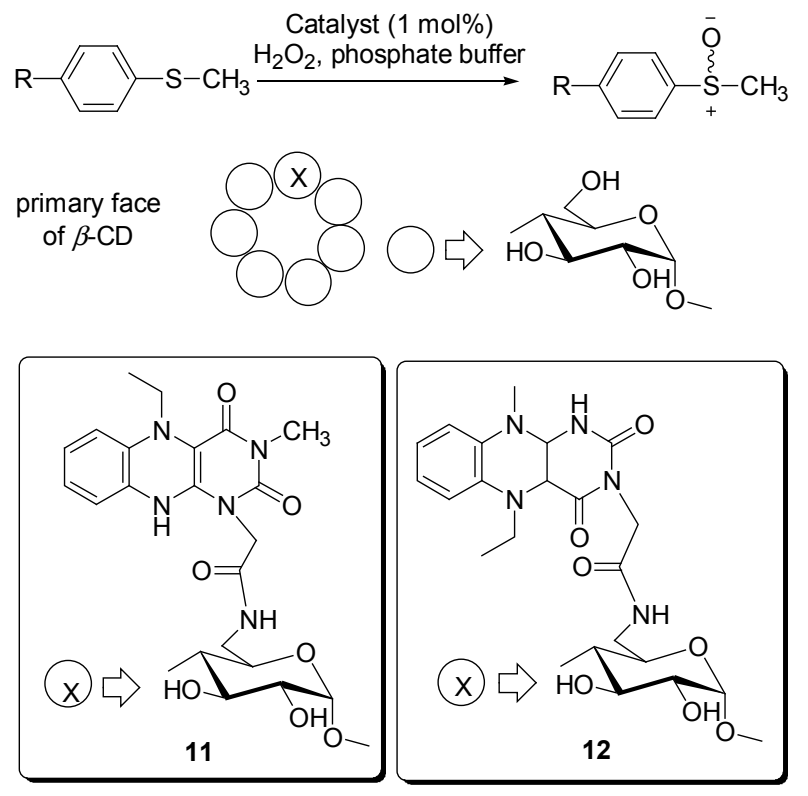

Scheme 10

起到立体选择的作用(Scheme 12) ${ }^{[105]}$. 黄素修饰的 $\beta$-环 糊精衍生物只需占反应总物质的量的 $0.2 \%$, 就可以使 产物的 $e e$ 值达到 $80 \%$. 实际上, 在溶液中, 环糊精手性 空腔的模板效应常常与疏水效应等共同作用, 最终完成 催化反应. 比如加成反应中, $\beta$-环糊精催化烯烃和水的 马氏加成反应合成对应的醇 ${ }^{[106]}$, 得到中等的 $e e$ 值 $32 \%$, 产率在 $14 \% \sim 0 \%$ 不等. 其中, $\beta$-环糊精空腔在反应中就 同时起到了逆相转移催化剂的作用, 这可能是为什么很 多溶液相的反应同样可以实现较高立体选择性的原因. 而在环糊精自身结构的氧化反应中, 空腔对结果立体选 择性的控制取决于空腔与氧化剂的包合强度, 这一过程 则完全的依赖于溶剂对包合强度的调节 ${ }^{[107]}$.

可见固相与液相反应条件对环糊精手性空腔模板 效应的影响非常复杂, 还有待继续深入研究.

\subsubsection{3 其他类型立体选择性反应举例}

$\beta$-环糊精/过硫酸氢钾体系是一种非常高效的氧化 试剂, 可以实现烯烃的环氧化反应. 例如, 利用该体系 对 $(S)$-萜烯进行环氧化反应发现 ${ }^{[108]}$, 如果使用天然 $\beta$-环 糊精, 更有利于反式产物的生成; 而如果选择丙酮酰 基- $\beta$-环糊精, 则有利于顺式产物的生成. 并且, 顺式和 反式产物的比例还与与萜烯分子上的取代基团的大小 有关. 位阻小的取代基有利于顺式产物的生成. $\beta$-环糊 精/过硫酸氢钾/丙酮体系对苯乙烯的不对称环氧化反应 中 $^{[109]}$, 苯乙烯环氧化的光学产率最高可达到 $29 \%$. 如 果, 利用单-2-丙酮基 $\beta$-环糊精/过硫酸氢钾体系 ${ }^{[110]}$ 也对 苯乙烯进行不对称环氧化, 在单-2-丙酮基 $\beta$-环糊精、过 硫酸氢钾、 $\mathrm{NaHCO}_{3}$ 和苯乙烯的物质的量比为 $2: 6$ : $12 ： 1$, 反应进行 $5 \mathrm{~h}$ 后, $e e$ 值可达到 $13 \%$.
再如, $\beta$-环糊精衍生物与钉的配合物可以用于催化 芳酮的不对称还原反应 ${ }^{[11]}$. 该反应中，产物的对映选 择性好, ee 值可高达 $97 \%$. 环糊精改造的膦配体与金属 铑配位作催化剂, 可用于催化 $\alpha$-醋胺肉桂酸、 $\alpha$-醋胺丙 烯酸、衣康酸及其甲酯的不对称氢化还原反应, $e e$ 值最 高可达 92\% ${ }^{[112 \sim 114]}$.

如果将小分子催化剂(4S)-苯氧基-(S)-脯氨酸固定 在 $\beta$-环糊精空腔内部 ${ }^{[115,116]}$, 可用于丙酮与邻硝基苯甲 醛的不对称羟醛反应, $e e$ 值可达到 $83.4 \%$.

利用主面胺基全取代的 $\beta$-环糊精的手性空腔可以 高立体选择性地实现硝基甲烷与含有不同取代基的醛 类化合物在乙腈-水混合体系(体积比 $1: 1$ )中的 Henry 反应. 该反应温度 $-20{ }^{\circ} \mathrm{C}$, 反应时间 $7 \mathrm{~h}$, 产率达到 $99 \%$, ee 值为 $99 \%$ (Scheme 11) ${ }^{[117]}$. 并且, 该 $\beta$-环糊精衍 生物还可以进一步实现以硝基乙烷和含有不同取代基 的醛类化合物为底物的 Henry 反应的非对映选择性, 顺/ 反选择性高达 99：1, 同时顺式产物的 $e e$ 值保持在 $99 \%$ (Scheme 11). 主面全氨基取代的 $\beta$-环糊精可以控制水相 中硝基甲烷/硫醇对反式查尔酮 Michael 加成反应的立体 选择性(Scheme 12 $)^{[118]}$. 前者的产率最高可达 $100 \%$, e.e. 值最高达 $87 \%$; 后者的产率最高可达 $100 \%, e e$ 值最高达 $60.6 \%$. 实际上, 使用手性修饰环糊精衍生物对有机反 应进行立体选择性的控制就是一种非常直接和有效的 方法. 例如, 在 Tsuji-Trost 反应中(Scheme 13) $)^{[119]}$, 分别 使用含苯基膦的环糊精对映异构体 13 和 14 与氯化钯配 位进行反应，所得对映异构体产物的产率分别为 77\%和 $78 \%, e e$ 值为 $30 \%$. 而使用非手性的环糊精配体 15 则无 法得到具有对映异构体的产物, 且产率很低.

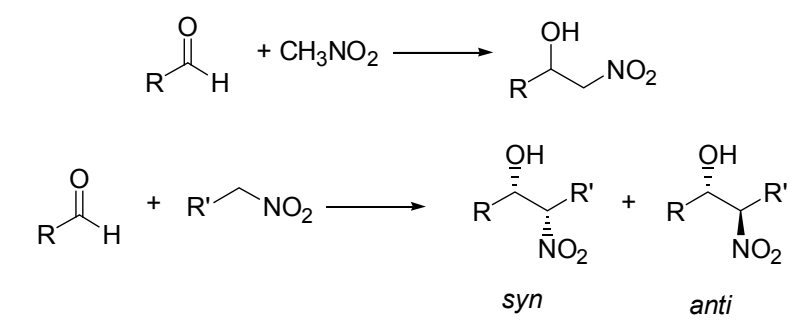

Scheme 11

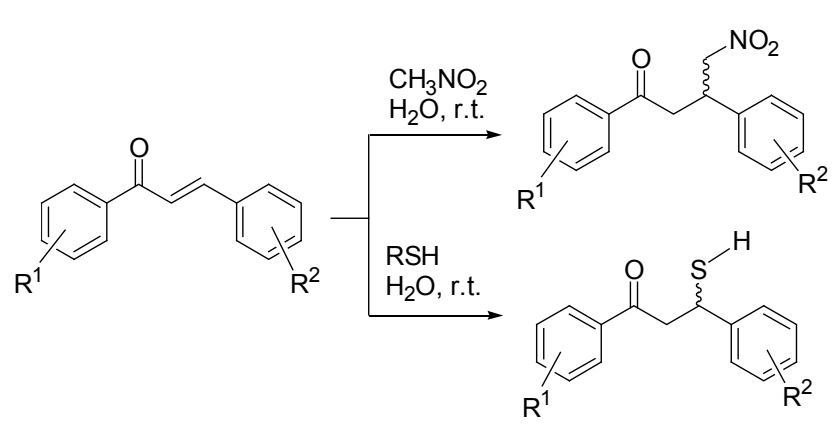

Scheme 12 


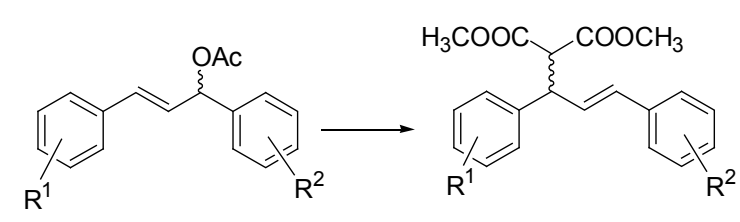

primary face of $\alpha-\mathrm{CD}$

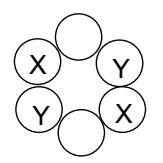

13

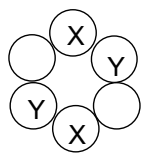

14

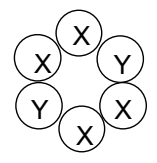

15
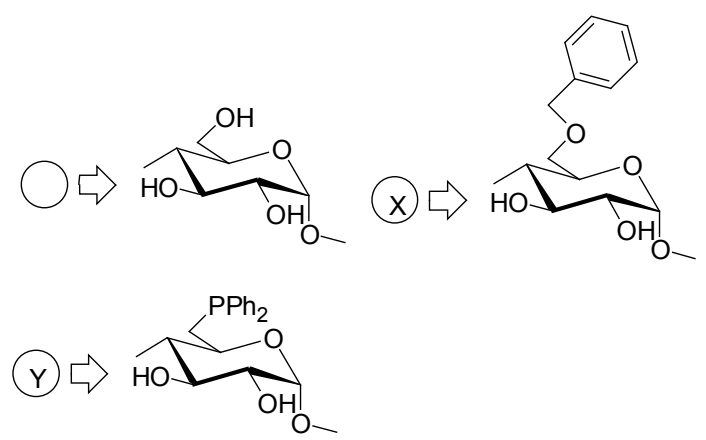

Scheme 13

\section{2 动态模板法}

模板在对反应进行 “限制” 的同时，自身也发生构 象、尺寸以及空间上的变化, 整个过程是一个动态发展 的过程.

\section{1 以桥连环糊精二聚体为模板}

相对于普通的环糊精衍生物来说, 桥连环糊精二聚 体因含有邻近的两个环糊精亚结构单元而具有协同作 用, 所以有非常强的与客体分子的包合作用及分子选择 性 ${ }^{[120]}$. 根据桥连环糊精二聚体中两个环糊精亚结构间 桥链的数目, 一般可将其分为单桥连环糊精二聚体和双 桥连环糊精二聚体 ${ }^{[21,122]}$. 目前, 用来作为有机反应模 板的桥连环糊精二聚体主要是单桥连二聚体.

桥连环糊精二聚体在有机反应中的模板作用主要 还是利用其所含的两个刚性空腔结构. 与普通的环糊精 及其衍生物类似, 桥连环糊精二聚体空腔的模板效应仍 然是依赖于其与底物或者产物分子的包合作用. 但是所 不同的是, 由于桥链的存在, 桥连环糊精二聚体与底物 或者产物分子的包合作用与其所含两个刚性空腔的空 间取向密切相关, 既链的存在限制了环糊精空腔空间取 向的自由度 ${ }^{[123,124]}$. 例如 ${ }^{[125]}$, 桥连环糊精二聚体 $\mathbf{1 6}$ (Scheme 14) 就与非线性的客体分子金莲橙 000 二号具 有很强的包合作用, 而二聚体 17 则与线形的甲基橙分 子有很不错的包合作用. 因此, 以二聚体 16 为反应模
板，常常得到非线性的产物分子.二吲哚啉-3,3'-二酮 (indigo)和二吲哚啉-2',3-二酮(indirubin)分别产生于两个 竞争反应, 既 $1 H$-吲哚-3-醇(indoxyl anion)之间的氧化二 聚反应及其与反应的氧化副产物 $1 H$-吲哚啉-2,3-二酮 (isatin)的缩合反应(Scheme 14). 当没有任何模板存在或 者只有 $\beta$-环糊精存在时, 这个竞争反应偏向于生成二吲 哚啉-3,3'二酮. 而当二聚体 16 存在时 ${ }^{[125,126]}$, 反应向生 成二吲哚啉-2'3-二酮的方向进行. 如果是在 $\mathrm{pH}$ 为 10.0 的条件下, 产物中二吲哚啉-2',3-二酮的含量甚至可以 是二吲哚啉-3,3'-二酮含量的 30 倍以上. 而如果采用桥 链稍长的二聚体 17 或者 18 , 反应对产物二吲哚啉- $2^{\prime}, 3-$ 二酮的选择性就会下降, 产物二吲哚啉-3,3'-二酮和二 吲哚啉-2',3-二酮的产量大体相等. 因此, 二聚体 16 中 两个环糊精空腔相对的空间取向在反应中发挥了关键 作用 ${ }^{[124]}$, 对产物二吲哚啉-3,3'-二酮的生成起到了限制 性作用; 而更倾向于包合底物 $1 H$-吲哚-3-醇和 $1 H$-吲哚 啉-2,3-二酮, 得到非线性的产物二吲哚啉-2',3-二酮.

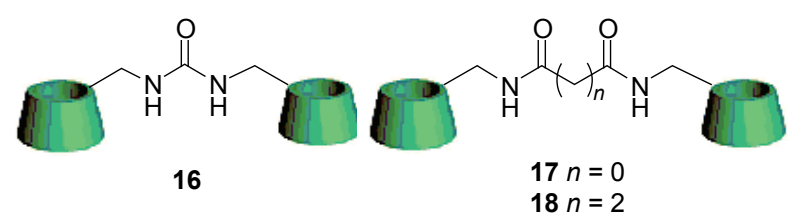<smiles>[R]c1c[nH]c2ccccc12</smiles>

$\mathrm{R}=\mathrm{O}^{-}$or $\mathrm{OAc}$ indoxyl anion<smiles>O=C1/C(=C2/Nc3ccccc3C2=O)Nc2ccccc21</smiles>

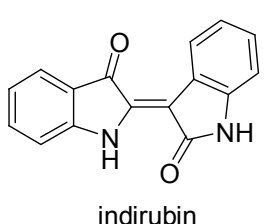

indirubin<smiles>O=C1Nc2ccccc2C1=O</smiles>

isatin

Scheme 14
基于强的包合作用(包合常数达到 $10^{9} \mathrm{~L} \cdot \mathrm{mol}^{-1}$ ) 和空 间构象上的限制, $\beta$-环糊精二聚体 19 21 可以作为模 板，控制性地合成脂溶性的金刚烷铼配体，使其选择性 地得到一种空间构型的配体产物(Scheme 15$)^{[127]}$. $\beta$-环 糊精二聚体存在时，在 $0{ }^{\circ} \mathrm{C}$ 条件下，反应 $1 \mathrm{~h}$, 金刚烷铼 配体的产率达到 $96 \%$, 且全部为反式构型(trans), ee 值 为 $100 \%$. 在没有 $\beta$-环糊精二聚体时, 这是不可实现的. 而在母体 $\beta$-环糊精存在时，也只有通过延长反应时间和 提高反应温度达到类似效果. 可见, $\beta$-环糊精二聚体作 为模板选择性合成配体反应的高效性.

锌(II)离子可以与 $\beta$-环糊精二聚体形成配合物 22 (Scheme 16) ${ }^{[128]}$, 它可以催化酯的水解. 在水解过程中, 


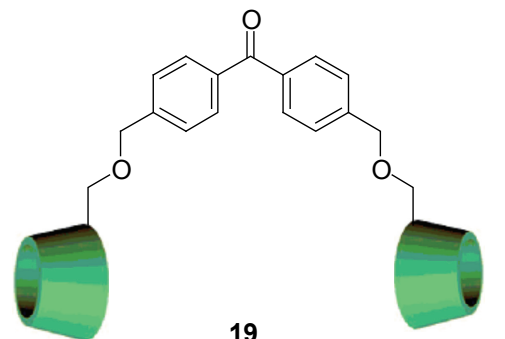

19

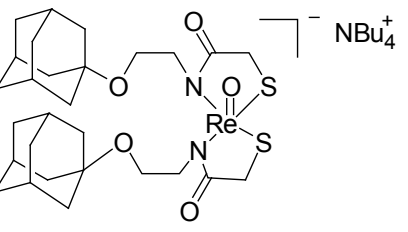

cis
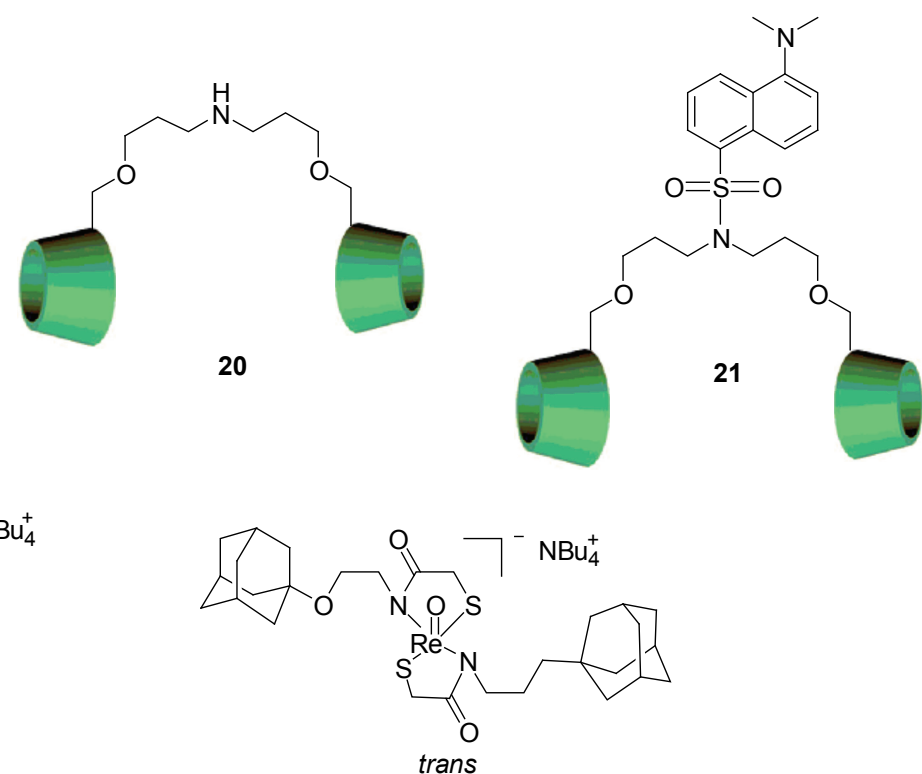

Scheme 15

由于空间构象等因素, 配合物中的两个环糊精空腔可以 作为模板, 对水解底物进行选择. 比如, 在对双 (4-硝 基-苯基)甲酸酯(BNPC)和 4-硝基-苯基乙酸酯(NA)的水 解反应中, 该配合物的存在使 BNPC 的水解速度提高了 $3.89 \times 10^{4}$ 倍, 而 NA 的水解速度只提高了 42 倍. 这主要 是由于 BNPC 与环糊精二聚体更匹配, 使得催化反应更 高效. 类似的, 使用该配体对双(4-硝基-苯基)磷酸酯 (BNPP) 的催化水解反应中, 其二级反应速率常数 (second-order rate constant, $K_{\text {cat }}$ ) 达到 $9.9 \times 10^{-4} \mathrm{~L}^{-} \mathrm{mol}^{-1}$ • $\mathrm{s}^{-1}$, 这在已报道的基于锌(II)离子的催化酯水解反应中 是最高的. 铜(II)离子与 $\beta$-环糊精二聚体的配合物 23 也 有类似的对水解反应的催化效果 (Scheme 16) ${ }^{[129]}$.

系列环糊精二聚体 24 26 被用来催化 $p$-硝基苯基 甲氧基二乙氧基乙酸酯的水解反应(Scheme 17$)^{[130]}$. 在
初始速率和底物浓度的关系图中发现，只有二聚体 $\mathbf{2 4}$ 可以给出一条 $\mathrm{S}$ 形曲线, 而另外两种二聚体仅给出了一 条简单的双曲线. 这表明二聚体 24 对该反应具有协同 催化效果, 其二级反应速率常数达到了 $1.05 \times 10^{-2}$ $\mathrm{L} \cdot \mathrm{mol}^{-1} \cdot \mathrm{s}^{-1}$. 其原因可能是二聚体 24 中含有 2 个 $\beta$-环 糊精, 其空腔的体积模板效应使其同反应底物形成 1 : 2 的包合物，使得水解反应更加高效. 由于协同效应和 良好的传质性能，通过 click 反应得到的 $\beta$-环糊精二聚 体 27 29 对 Tsuji-Trost 反应也有很好的模板控制作用 (Scheme 18) ${ }^{[131]}$.

\section{2 以环糊精自组装体系为模板}

环糊精自组装体系作为有机反应模板的研究只是 近几年才刚刚起步的. 目前, 这方面的研究主要是将环

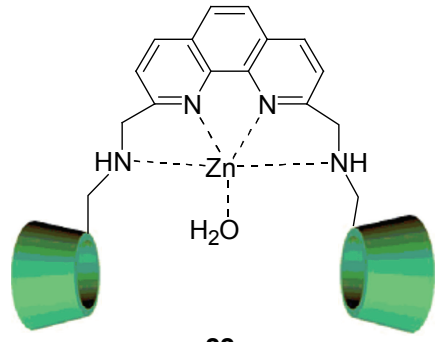

22

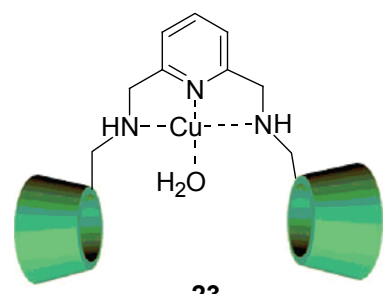

23<smiles>O=[N+]([O-])c1ccc(OP(=O)(O)Oc2ccc([N+](=O)[O-])cc2)cc1</smiles>

BNPP<smiles>O=C(Oc1ccc([N+](=O)[O-])cc1)Oc1ccc([N+](=O)[O-])cc1</smiles>

BNPC<smiles>CC(=O)Oc1ccc([N+](=O)[O-])cc1</smiles>

NA

Scheme 16 


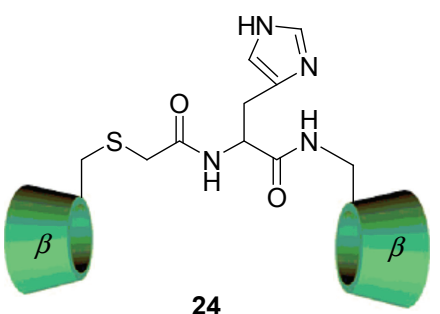

24

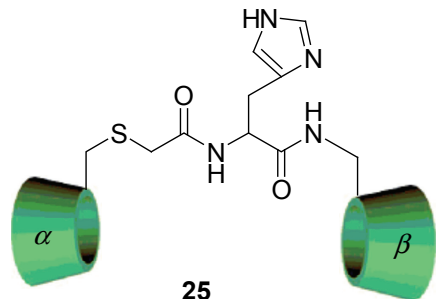

25

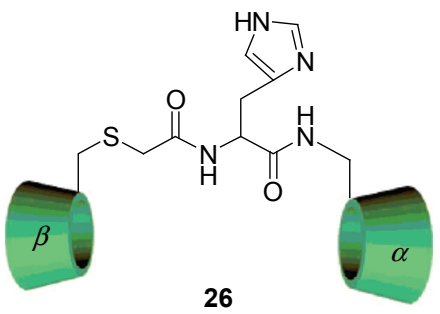

26<smiles>COCCOCCOCC(=O)Oc1ccc([N+](=O)[O-])cc1</smiles>

Scheme 17

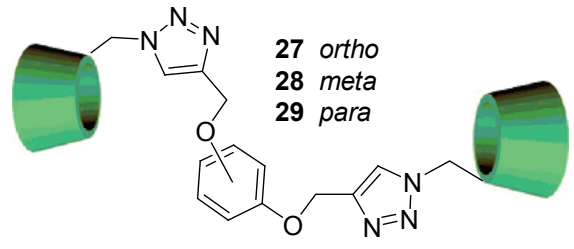

Scheme 18

糊精结构与表面活性剂体系混用，使其共同发挥模板作 用. 众所周知, 表面活性剂所形成的囊泡、胶束以及微 乳液等常具有对有机反应催化的作用 ${ }^{[132]}$. 当向这些聚 集体系中加入环糊精结构单元时, 由于环糊精空腔具有 包合表面活性剂的能力, 因此可以调节原有表面活性剂 体系的表面张力等性质. 按照 “超分子环糊精两亲分子” 理论的划分, 这种环糊精与表面活性剂的包合物属于第 二类 “超分子环糊精两亲分子” ${ }^{[133]}$, 可以根据包合物自 身的 “憎溶剂” 性质，调整自组装体的形貌，对反应的 底物和产物具有选择性, 最终实现对有机反应模板调节
的作用(图 4).

例如, 环糊精结构就曾与咪唑类表面活性剂结合, 共同调节热控制的烯烃氢甲酰化反应(Scheme 19) ${ }^{[134]}$. 常温下, $\beta$-环糊精可以与咪唑类表面活性剂的烷基链部 分形成包合物; 而温度达到 $80{ }^{\circ} \mathrm{C}$ 时, 咪唑盐就会形成 胶束. 环糊精与咪唑类表面活性剂共同作为反应的模 板，有利于反应结束后，低温条件下模板体系与产物的 分离. 这就大大提高了产率，使产率达到 73\% 100\%; 同时，增加了反应的区域选择性，线形产物与枝形产物 比 $80 \% \sim 96 \%$. 这个结果与前文中提到的、单纯环糊精 参与的氢甲酰化反应相比, 转化率和产物的专一性都有 很大的提高(Eq. 1).

imidazolium surfactants

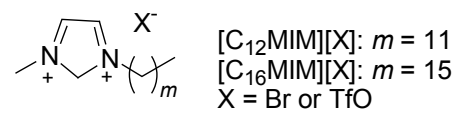

Scheme 19
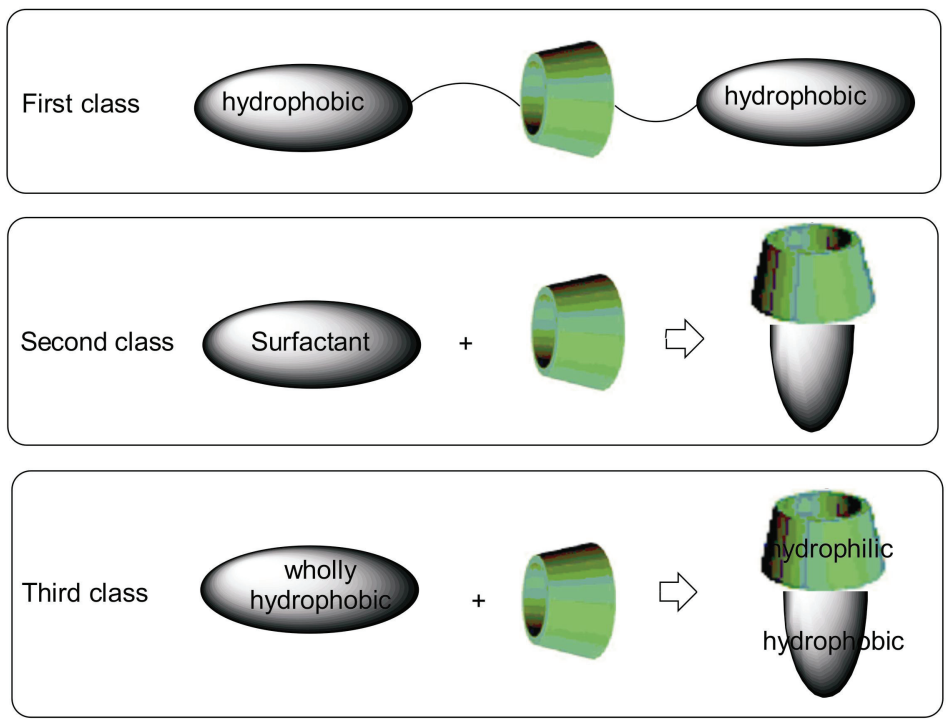

图 4 超分子环糊精表面活性剂

Figure 4 Supramolecular cyclodextrins amphiphiles (SCS) 
环糊精也常常被应用到聚合物的水相合成中 ${ }^{[135,136]}$, 其刚性结构大多只是起到相转移催化剂的功能. 但是, 当环糊精与表面活性剂体系联合使用时，环糊精与表面 活性剂就会共同发挥模板效应. 例如, 在毛海胆形聚苯 胺的合成中, 就采用了表面活性剂和 $\beta$-环糊精的混合体 系作为模板 ${ }^{[137]}$. 首先, 使用阳离子表面活性剂十二烷 基三甲基氯化铵(dodecyl trimethyl ammoniumchloride, DoTAC) 在水溶液中形成胶束, 然后将 $\beta$-环糊精与苯胺 的包合物加入该胶束溶液中. 在主体浓度过量的情况 下, $\beta$-环糊精与苯胺的包合物会进入胶束的疏水空腔或 者停滞在胶束的壁层. 使用过硫酸铵 (ammonium persulfate, APS)作引发剂, 便可以得到处于胶束中的 $\beta$-环 糊精与聚苯胺的包合物. 之后, 利用三氯化铁为氧化剂, 使阳离子表面活性剂的头基发生相互作用, 降低了第二 临界胶束浓度(critical micelle concentration II, CMC(II)), 使得原有的球形胶束向棒形胶束转化. 与此同时, 当 $\beta$ 环糊精过量时, 胶束核内的 $\beta$-环糊精与聚苯胺包合物会 逐渐替代掉处于壁层的 $\beta$-环糊精与苯胺的包合物, 最终 得到毛海胆形聚苯胺. 又如, 在制备具有规则形貌的聚 吡咯的过程中，第二类 “超分子环糊精两亲分子” (图

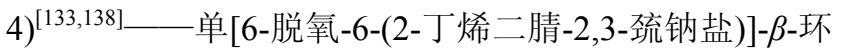
糊精与甲基橙的包合物(Eq. 3), 或者 $\beta$-环糊精与酸性红 $\mathrm{G}$ 的包合物(Eq. 4)一的存在非常重要, 它们可以自组 装成对称的条带型通道 ${ }^{[139]}$ 或者规则的膜片结构 ${ }^{[140]}$, 用 作模板, 以引导聚吡咯形成对称的辐射型形貌或者规则 的六角片状形貌。
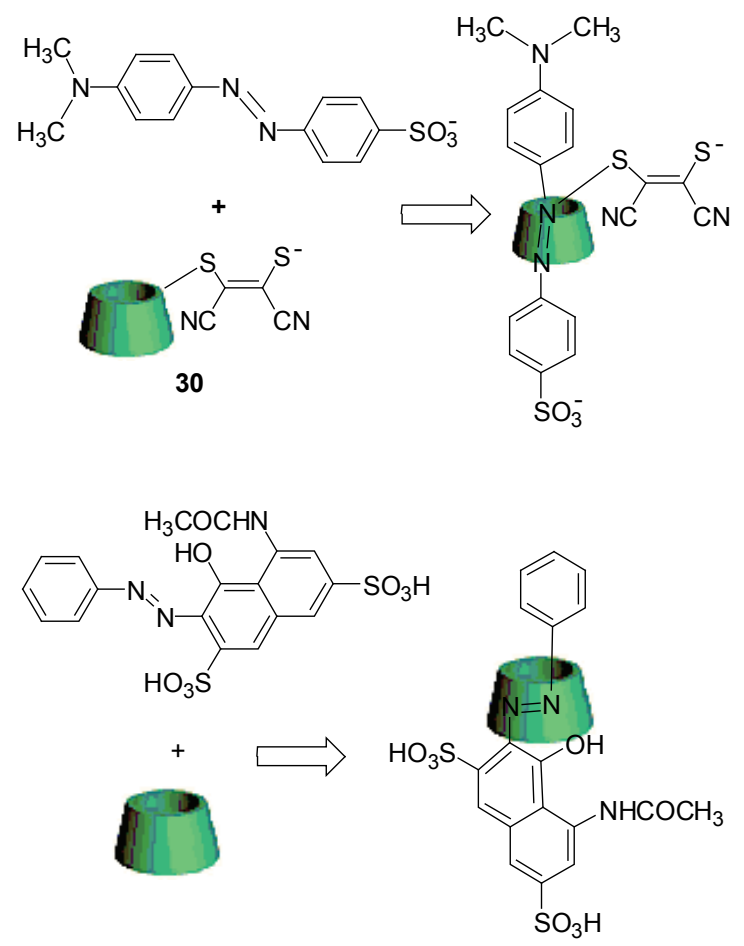

实际上，疏水烷基链共价键修饰的环糊精衍生 物，作为第一类 “超分子环糊精两亲分子” (图 4)也可以 在有机反应中发挥模板作用。例如，同样是烯烃氢甲酰 化反应，以铑为催化剂的 1-癸烯的两相氢甲酰化反应 中, 长烷基链修饰的 $\beta$-环糊精(疏水链长为七个碳)存在 下，转化率可以达到 $26 \% \sim 56 \%$, 而区域选择性更是可 以达到 $89 \% \sim 98 \%$, 线形与直形产物比最高可达到 $2.8^{[141]}$. 按照第一类 “超分子环糊精两亲分子” 的理 论 ${ }^{[133]}$, 当疏水性烷基链长于六碳时，该分子很容易在 水溶液中形成类似于胶束或囊泡的自组装聚集体. 但 是，该反应中，烷基修饰环糊精的模板作用机理是否与 其自组装形貌的形成有关尚需进一步研究 ${ }^{[132]}$.

\section{3 总结与展望}

综上所述，不难发现，环糊精及其衍生物不但可以 使用自身稳定的刚性结构，例如主、次面和手性疏水空 腔作为静态模板控制反应的进程以及产物的形状、大 小、手性和异构体等; 而且, 桥连环糊精二聚体、修饰 环糊精以及环糊精包合物形成的自组装结构也可以作 为动态模板控制产物的形貌等因素(图 3). 值得注意的 是, 一方面, 近年来, 基于环糊精模板的有机反应还在 不断地开发和研究中; 另一方面，环糊精动态模板的开 发为有机化学家和超分子化学家控制性地进行反应提 供了一种新的思路. 因此, 基于环糊精为模板的有机反 应的研发工作具有非常广阔的前景.

结合近年来相关领域的研究现状以及环糊精模板 法自身的特点，对该领域做以下几个方面的展望. (1)利 用环糊精主次面的模板效应，可以考虑合成具有更复杂 的拓扑结构的新型分子. 近年来, 以 Stoddart 等 ${ }^{[142]}$ 为代 表的超分子化学家正在致力于研究和制备具有 “锁式” 结构的新型分子. 这类新型分子对于丰富超分子化学和 有机化学的化合物种类非常重要, 对这类分子的研究也 已逐渐成为一个相对独立的新兴交叉领域. (2)对于利用 环糊精刚性的手性空腔为模板的有机反应，可以更多地 考虑与金属配合物相结合. 环糊精络合金属离子更有利 于环糊精母体模拟天然酶的反应特点，使其在有机反应 中的催化效果更为明显和高效，模板效应更显著. 而且， 环糊精与金属配合物相结合的体系应用日益广泛，是环 糊精模板在有机合成中的重要发展方向. 值得一提的 是, 最近发展出了一种新型的环糊精与金属配位的刚性 自组装结构——环糊精金属-有机框架结构(metal-organic frameworks, MOF ${ }^{[143]}$. 它具有结构稳定、制备简单 的特点, 可以考虑将其应用到环糊精模板法或者催化领 域. 此外，基于环糊精的有机和无机杂化材料也是一个 有价值的选择 ${ }^{[144]}$. (3)双桥连环糊精体系中 ${ }^{[122]}$, 两个环 
糊精分子间通过更加刚性的桥链结构连接, 环糊精的空 间结构相对固定. 可以考虑将这种既具有相对刚性结 构, 又具有两个空腔协同效应的体系应用的环糊精模板 法中. (4)关于环糊精衍生物及其包合物形成的自组装体 系, 如胶束、囊泡等, 目前的实例还很有限 ${ }^{[132]}$. 固然, 该领域存在研究上的难度, 但是, 如果能够成功地把环 糊精的疏水空腔模板与囊泡的双层结构相结合, 那么这 不但具有有机合成方法学上的突破意义, 更是给人类模 拟细胞催化奠定了基础 ${ }^{[145]}$. 此外, 环糊精的分子印记 (molecular imprinting)技术, 作为一种自组装形式, 也是 环糊精模板法下一步发展的重要方向. (5)环糊精模板控 制体系的多功能化也是该领域的一个新的思路. 例如, 可以考虑将分子机器(开关)等新技术引入到模板法中, 以实现对模板自身的控制. 例如, 利用环糊精包合物可 以作为反应的响应开关 ${ }^{[146]}$, 既环糊精空腔发挥模板作 用的同时还可以作为反应的开关, 控制反应的进程. 这 样不但可以在三维空间上控制反应底物的结构, 还可以 在时间轴上控制反应的发生与否, 最终实现对反应的 “四维” 控制. (6)在环糊精模板法的理论层面, 下一步的 研究需要更深入地探讨其发生和作用的机理, 给出更加 科学合理的理论模型. 这对于指导未来基于环糊精模板 法的有机合成反应至关重要. 总之, 基于环糊精模板法 的有机合成前景广阔, 亟待有机化学、超分子化学、胶 体与界面化学等领域的科研工作者共同努力, 合作开 发.

致谢 感谢中华人民共和国教育部及国家留学基金委 (Chinese Scholarship Council, CSC) “建设高水平大学” 公派留学项目对本文第一作者在美国西北大学访学期 间的资助.

\section{References}

[1] Szejtli, J. Chem. Rev. 1998, 98, 1743.

[2] Lecourt, T.; Mallet, J. M.; Sinay, P. Eur. J. Org. Chem. 2003, 4553.

[3] Liu, Y.; You, C.-C.; Zhang, H.-Y. Supramolecular Chemistry: Molecular Recognition and Assembly of Synthetic Receptors, Nankai University Press, Tianjin, 2001 (in Chinese). (刘育, 尤长城, 张衡益, 超分子化学—合成受体的分子识别 与组装, 南开大学出版社, 天津, 2001.)

[4] Weiner, W.; Winkler, J.; Zimmerman, S. C.; Czarnik, A. W.; Breslow, R. J. Am. Chem. Soc. 1985, 107, 4095.

[5] Desper, J. M.; Breslow, R. J. Am. Chem. Soc. 1994, 116, 12081.

[6] Marinescu, L.; Bols, M. Trends Glycosci. Glycotechnol. 2009, 21, 309.

[7] Breslow, R. Acc. Chem. Res. 1995, 28, 146.

[8] Zhao, M.; Hao, A.; Wang, J. Chemistry 2005, 68, w086 (in Chinese). (赵明刚, 郝爱友, 王建英, 化学通报, 2005, 68, w086.)

[9] Zhao, M.; Hao, A. Chemistry 2006, 69, w001 (in Chinese). (赵明刚, 郝爱友, 化学通报, 2006, 69, w001.)
[10] Canipelle, M.; Landy, D.; Fourmentin, S. J. Inclusion Phenom. Macrocyclic Chem. 2011, 69, 349.

[11] Kumar, A.; Tripathi, V. D.; Kumar, P. Green Chem. 2011, 13, 51.

[12] Sun, H.-Y.; Hao, A.-Y.; Yin, M.-J.; Zhang, H.-C.; Shen, J. Prog. Chem. 2008, 20, 1694 (in Chinese).

(孙宏元, 郝爱友, 尹明静, 张华承, 申健, 化学进展, 2008, 20, 1694.)

[13] Sun, T.; Sun, T.; Shen, J. Chin. J. Org. Chem. 2009, 29, 681 (in Chinese).

(孙涛, 郝爱友, 申健, 有机化学, 2009, 29, 681.)

[14] Jin, T.; Wang, A.; Zhang, J.; Zhao, R.; Liu, B. Chin. J. Org. Chem. 2006, 26, 1723 (in Chinese).

(靳通收, 王爱卿, 张建设, 赵瑞巧, 刘利宾, 有机化学, 2006, 26, 1723.)

[15] Rideout, D. C.; Breslow, R. J. Am. Chem. Soc. 1980, 102, 7816.

[16] Kim, S. P.; Leach, A. G.; Houk, K. N. J. Org. Chem. 2002, 67, 4250.

[17] Naskar. S.; Saha, P.; Paira, R.; Paira, P.; Hazra, A.; Sahu, K. B.; Banerjee, S.; Mondal, N. B. J. Chem. Res., Synop. 2009, 174.

[18] Ji, H.; Huang, L.; Shi, D.; Zhou, X. Chin. J. Org. Chem. 2008, 28, 2072 (in Chinese).

(纪红兵, 黄丽泉, 石东坡, 周贤太, 有机化学, 2008, 28, 2072.)

[19] Breslow, H. Acc. Chem. Res. 1991, 24, 159.

[20] Xiao, Y.-M.; Fu, B; Li, N.; Tan, Z.-H. Prog. Chem. 2005, 17, 692 (in Chinese).

(肖玉梅, 傅滨, 李楠, 覃兆海, 化学进展, 2005, 17, 692.)

[21] Saito, R.; Okuno, Y.; Kobayashi, H. J. Polym. Sci., Part A: Polym. Chem. 2001, 39, 3539.

[22] Saito, R.; Kobayashi, H. Macromolecules 2002, 35, 7207.

[23] Saito, R. Polymer 2008, 49, 2625.

[24] Saito, R.; Yamaguchi, K. Macromolecules 2003, 36, 9005.

[25] Saito, R.; Yamaguchi, K. Macromolecules 2005, 38, 2085.

[26] Sait o, R.; Kobayashi, H. J. Inclusion Phenom. Macrocycl. Chem. 2002, 44, 303.

[27] Saito, R.; Yamaguchi, K. J. Polym. Sci., Part A: Polym. Chem. 2005, 43, 6262 .

[28] Saito, R.; Yamaguchi, K.; Hara, T.; Saegusa, C. Macromolecules 2007, 40, 4621.

[29] Rossi, L. I.; Rossi, R. H. Appl. Catal., A 2004, 267, 267.

[30] Kinen, C. O.; Rossi, L. I.; Rossi, R. H. Appl. Catal. A 2006, 312, 120.

[31] Ji, H.-B.; Shi, D.-P.; Shao, M.; Li, Z.; Wang, L-F. Tetrahedron Lett. 2005, 46, 2517.

[32] Tan, K.-Y.; Su, G.-F.; Rao, W.-P.; Tan, G.-M. Chin. J. Org. Chem. 2006, 26, 1623 (in Chinese).

(覃开云, 苏桂发, 饶万平, 谭光明, 有机化学, 2006, 26, 1623.)

[33] Surendara, K.; Krishnaveni, N. S.; Reddy, M. A.; Nageswar, Y. V. D.; Rao, K. R. J. Org. Chem. 2003, 68, 9119.

[34] Krishnaveni, N. S.; Surendra, K.; Nageswar, Y. V. D.; Rao, K. R. Synthesis 2003, 1968.

[35] Anton, L. M.; Dimitri, A. S.; Tatyana, Y.; Filippova, A. Y. Z.; Edward, A. K. Ind. Eng. Chem. Res. 2005, 44, 8644.

[36] Fenger, T. H.; Bols, M. J. Inclusion Phenom. Macrocyclic Chem. 2011, 69, 397.

[37] Akkilagunta, V. K.; Reddy, V. P.; Kakulapati, R. R. Synlett 2010, 2517.

[38] Bjerre, J.; Bols, M. J. Inclusion Phenom. Macrocyclic Chem. 2011, $69,417$.

[39] Marinescu, L. G.; Doyaguez, E. G.; Petrillo, M.; Fernandez-Mayoralas, A.; Bols, M. Eur. J. Org. Chem. 2010, 157.

[40] Fenger, T. H.; Bjerre, J.; Bols, M. Chem. Biochem. Eng. $Q$ 2009, 23, 2494. 
[41] Ji, H.-B.; Huang, L.-Q.; Wu, B. Acta Chim. Sinica 2009, 67, 910 (in Chinese).

(纪红兵, 黄丽泉, 吴特, 化学学报, 2009, 67, 910.)

[42] Fenger, T. H.; Marinescu, L. G.; Bols, M. Org. Biomol. Chem. 2009, 7, 933.

[43] Machut-Binkowski, C.; Legrand, F. X.; Azaroual, N.; Tilloy, S.; Monflier, E. Chem. Eur. J. 2010, 16, 10195.

[44] Bjerre, J.; Bols, M. Eur. J. Org. Chem. 2010, 3487.

[45] Basilio, N.; Rio, L. G.; Moreira, J. A.; Pessego, M. J. Org. Chem. 2010, 75,848

[46] Sridhar, R.; Srinivas, B.; Madhav, B.; Reddy, V. P.; Nageswar, Y. V. D.; Rao, K. R. Can. J. Chem. 2009, 87, 1704.

[47] Zhang, M.; Gao, Y.; Hu, R. Z.; Shi, Z. Chin. Chem. Lett. 2008, 19, 1139.

[48] Krishnaveni, N. S.; Surendra, K.; Reddy, M. S.; Negeswar, Y. V. D.; Rao, K. R. Adv. Synth. Catal. 2004, 346, 395.

[49] Kuppusamy, K.; Pitchumani, K. Tetrahedron Lett. 2010, 51, 3312.

[50] Murthy, S. N.; Madhav, B.; Reddy, V. P.; Nageswar, Y. V. D. Tetrahedron Lett. 2010, 51, 3649.

[51] Murthy, S. N.; Madhav, B.; Kumar, A. V.; Rao, K. R.; Nageswar, Y. V. D. Helv. Chim. Acta 2009, 92, 2118

[52] Murthy, S. N.; Madhav, B.; Kumar, A. V.; Rao, K. R.; Nageswar, Y. V. D. Tetrahedron, 2009, 65, 5251.

[53] Kumar, V. P.; Sridhar, R.; Srinivas, B.; Narender, M.; Rao, K. R. Can. J. Chem. 2008, 86, 907.

[54] Bricout, H.; Carbon, L.; Bormann, D.; Monflier, E. Catal. Today 2001, 66, 355

[55] Lidia, S.; Liu, J.; Kaifer, A. E. Langmuir 2003, 19, 483.

[56] Nakamura, A.; Inoue, Y. J. Am. Chem. Soc. 2005, 127, 5338.

[57] Torque, C.; Bricoat, H.; Hapiot, F.; Monflier, E. Tetrahedron 2004, $60,6487$.

[58] Torque, C.; Sueur, B.; Cabou, J.; Bricout, H.; Hapiot, F.; Monflier, E. Tetrahedron 2005, 61, 4811.

[59] Breslow, R.; Winnik, M. A. J. Am. Chem. Soc. 1969, 91, 3083.

[60] Palaniswamy, S.; Subramanian, A.; Kasi, P. Tetrahedron 2007, 63, 4959.

[61] Zhuang, X.-J.; Yuan, F.; Zhang, S.-S. J. Univ. Sci. Technol. 2004, 24, 24 (in Chinese). (庄新杰, 袁枫, 张书圣, 青岛科技大学学报, 2004, 24, 24.)

[62] Shiraishi, Y.; Tomita, H.; Fujiki, K.; Hirai, H. React. Funct. Polym. 1998, 36, 99 .

[63] Shiraishi, Y.; Toshima, N.; Kawamura, T.; Mihori, H.; Shirai, H.; Hirai, H. J. Mol. Catal. A: Chem. 1999, 139, 149

[64] Hirai, H. Polym. Adv. Techonl. 1997, 8, 666.

[65] Mathivet, T.; Méliet, C.; Castanet, Y.; Mortreux, A.; Caron, L.; Tilloy, S.; Monflier, E. J. Mol. Catal. A: Chem. 2001, 176, 105.

[66] Leclercq, L.; Hapiot, F.; Tilloy, S.; Ramkisoensing, K.; Reek, J. N. H.; Leeuwen, P. W. N. M.; Monflier, E. Organometallics 2005, 24, 2070 .

[67] Leclercq, L.; Sauthier, M.; Castanet, Y.; Mortreux, A.; Bricout, M. H.; Monflier, E. Adv. Synth. Catal. 2005, 347, 55.

[68] Legrand, F. X.; Sauthier, M.; Flahaut, C.; Hachani, J.; Elfakir, C.; Fourmentin, S.; Tilloy, S.; Monflier, E. J. Mol. Catal. A: Chem. 2009, 303, 72 .

[69] Leclercq, L.; Schmitzer, A. R. Organometallics 2010, 29, 3442

[70] Li, W. S.; Chung, W. S.; Chao, I. Chem. Eur J. 2003, 9, 951.

[71] Reddy, M. S.; Srinivas, B.; Sridhar, R.; Narender, M.; Rao, K. R. J. Mol. Catal. A: Chem. 2006, 255, 180.

[72] Surendra, K.; Krishnaveni, N. S.; Nageswar, Y. V. D.; Rao, K. R. J. Org. Chem. 2003, 68, 4994.

[73] Krishnaveni, N. S.; Surendara, K.; Narender, M.; Nageswar, Y. V. D.; Rao, K. R. Synthesis 2004, 501.
[74] Krishnaveni, N. S.; Surendra. K.; Rao, K. R. Adv. Synth. Catal. 2006, 348, 696.

[75] Somi, R. M.; Narender, M.; Rama, R. K. Tetrahedron Lett. 2005 , 46, 1299.

[76] Banu, H. S.; Pitchumani, K.; Srinivasan, C. Tetrahedron 1999, 55, 9601.

[77] Koodanjerl, S.; Pradhan, A. R.; Kaanumalle, L. S.; Ramamurthy, V. Tetrahedron Lett. 2003, 44, 3207.

[78] Komiyama, M.; Hirai, H. J. Am. Chem. Soc. 1983, 105, 2018.

[79] Ravichandran, R. J. Mol. Catal. A: Chem. 1998, 130, L205.

[80] Ueno, A. J. Am. Chem. Soc. 1991, 113, 7034.

[81] Nakamura, A.; Inoue, Y. J. Am. Chem. Soc. 2003, 125, 966.

[82] Yang, C.; Nakamura, A.; Fukuhara, G.; Origane, Y.; Mori, T.; Wada, T.; Inoue, Y. J. Org. Chem. 2006, 71, 3126

[83] Ikeda, H.; Nihei, T.; Ueno, A. J. Inclusion Phenom. Macrocyclic Chem. 2004, 50, 63.

[84] Ikeda, H.; Nihei, T.; Ueno, A. J. Org. Chem. 2005, 70, 1237.

[85] Ke, C.; Yang, C.; Mori, T.; Wada, T.; Liu, Y.; Inoue, Y. Angew. Chem., Int. Ed. 2009, 48, 6675.

[86] Yang, C.; Fukuhara, G.; Nakamura, A.; Origane, Y.; Fujita, K.; Yuan, D. Q.; Mori, T.; Wada, T.; Inoue, Y. J. Photochem. Photobiol., A 2005, 173, 375.

[87] Yang, C.; Nishijima, M.; Nakamura, A.; Mori, T.; Wada, T.; Inoue, Y. Tetrahedron Lett. 2007, 48, 4357.

[88] Yang, C.; Nakamura, A.; Wada, T.; Inoue, Y. Org. Lett. 2006, 8, 3005.

[89] Yang, C.; Mori, T.; Inoue, Y. J. Org. Chem. 2008, 73, 5786.

[90] Yang, C.; Ke, C.; Kahee, F.; Yuan, D. Q.; Mori, T.; Inoue, Y. Aust. J. Chem. 2008, 61, 565.

[91] Ke, C.; Yang, C.; Liang, W.; Mori, T.; Liu, Y.; Inoue, Y. New J. Chem. 2010, 34, 1323

[92] Yang, C.; Mori, T.; Origane, Y.; Ko, Y. H.; Selvapalam, N.; Kim, K.; Inoue, Y. J. Am. Chem. Soc. 2008, 130, 8574.

[93] Fukuhara, G.; Nakamura, T.; Yang, C.; Mori, T.; Inoue, Y. Org. Lett. 2010, 12, 3510 .

[94] Luo, L.; Liao, G. H.; Wu, X. L.; Lei, L.; Tung, C. H.; Wu, L. Z. J. Org. Chem. 2009, 74, 3506.

[95] Tong, L.-H.; Lu, R.-H.; Inoue, Y. Prog. Chem. 2006, 18, 533 (in Chinese).

(童林荟，鲁润华，井上佳久，化学进展, 2006, 18, 533.)

[96] Fukuhara, G.; Mori, T.; Wada, T.; Inoue, Y. J. Org. Chem. 2006, 71, 8233.

[97] Lu, R.; Yang, C.; Cao, Y.; Wang, Z.; Wada, T.; Jiao, W.; Mori, T.; Inoue, Y. Chem. Commun. 2008, 374.

[98] Lu, R.; Yang, C.; Cao, Y.; Tong, L.; Jiao, W.; Wada, T.; Wang, Z.; Mori, T.; Inoue, Y. J. Org. Chem. 2008, 73, 7695.

[99] Yang, C.; Mori, T.; Wada, T.; Inoue, Y. New J. Chem. 2007, 31, 697.

[100] Koodanjeri, S.; Joy, A.; Ramamurthy, V. Tetrahedron 2000, 56, 7003.

[101] Rao, K. S. S. P.; Hubig, S. M.; Moorthy, J. N.; Kochi, J. K. J. Org. Chem. 1999, 64, 8098

[102] Shailaja, J.; Karthikeyan, S.; Ramamurthy, V. Tetrahedron Lett. 2002, 43, 9335.

[103] Cyril, R.; Brian, C.; Mikael, B. Eur. J. Org. Chem. 2005, 13, 2734

[104] Gandofli, R.; Borrometi, A.; Romano, A.; Gago, J. V. S.; Molinari, F. Tetrahedron: Asymmetry 2002, 13, 2345.

[105] Mojr, V.; Herzig, V.; Budesinsky, M.; Cibulka, R.; Kraus, T. Chem. Commun. 2010, 46, 7599.

[106] Artur, R. A.; Iva, C.; Carla, R.; Luısa, M. F.; Ana, L.; Pedro, P. S. Tetrahedron 2005, 61, 11986

[107] Armspach, D.; Matt, D.; Toupet, L. Angew. Chem., Int. Ed. 2009, 48,4555 . 
[108] Chan, W. S.; Yu, W. Y.; Che, C. M.; Wong, M. K. J. Org. Chem. 2003, 68, 6576 .

[109] Deng, F.; Lan, Zh.-L.; Yin, D.-L.; Xiao, Z.-S. Chemistry 2006, 69, 362 (in Chinese). (邓芳, 兰支利, 尹笃林, 肖自胜, 化学通报, 2006, 69, 362.)

[110] Li, C.-H.; Lan, Z.-L.; Yin, D.-L.; Yu, H.-J. Ind. Catal. 2008, 16, 37 (in Chinese). (李传华，兰支利，尹笃林，于红杰，工业催化， 2008, 16, 37.)

[111] Schlatter, A.; Kunda, M. K.; Woggon, W. D. Angew. Chem. Int. Ed. 2004, 43, 6731.

[112] Yang, C. L.; Cheung, Y. K.; Yao, J. Z.; Wong, T. Y.; Jia, G. Organometallics 2001, 20, 424.

[113] Yang, C. L.; Wong, Y. T.; Li, Z. Y.; Krepinsky, J. J.; Jia, G. Organometallics 2001, 20, 5220.

[114] Wong, Y. T.; Yang, C. L.; Ying, K. C.; Jia, G. Organometallics 2002, $21,1782$.

[115] Shen, Z.-X.; Ding, Y.; Liu, Y.-H.; Zhang, Y.-W. Chin. J. Org. Chem. 2005, 25, 1113 (in Chinese). (沈宗旋, 丁一, 李明, 刘艳华, 张雅文, 有机化学, 2005, 25, 1113 )

[116] Shen, Z.-X.; Ma, J.-M.; Liu, Y.-H.; Jiao, C.-J.; Li, M.; Zhang, Y.-W. Chirality. 2005, 17, 556.

[117] Kanagaraj, K.; Suresh, P.; Pitchumani, K. Org. Lett. 2010, 12, 4070.

[118] Suresh, P.; Pitchumani, K. Tetrahedron: Asymmetry 2008, 19, 2037.

[119] Guieu, S.; Zaborova, E.; Bleriot, Y.; Poli, G.; Jutand, A.; Madec, D.; Prestat, G.; Sollogoub, M. Angew. Chem., Int. Ed. 2010, 49, 2314

[120] Liu, Y.; Chem, Y. Acc. Chem. Res. 2006, 39, 681.

[121] Zhang, H.-C.; Hao, A.-Y.; Shen, J. Chin. J. Org. Chem. 2008, 28, 954 (in Chinese). (张华承，郝爱友，申健，有机化学, 2008, 28, 954.)

[122] Zhang, H.-C.; Liu, Z.-N.; An, W.; Hao, A.-Y.; Sun, L.-Z. Chin. J. Org. Chem. 2010, 30, 1279 (in Chinese). (张华承, 刘召娜, 安伟, 郝爱友, 孙立臻, 有机化学, 2010, 30, 1279.)

[123] Easton, C. J.; Lincoln, S. F.; Barr, L.; Onagi, H. Chem. Eur. J. 2004, 10, 3120.

[124] Blenke, C.; Silva, V. J. D.; Junqueira, M. A. G.; Almeida, W. B. D.; Santos, H. F. D. J. Mol. Struct. THEOCHEM 2007, 809, 95.

[125] Easton, C. J.; Harper, J. B.; Lincoln, S. F. New J. Chem. 1998, 1163.

[126] Harper, J. B.; Easton, C. J.; Lincoln, S. F. Tetrahedron Lett. 2003, 44,5815 .
[127] Bommel, K. J. C.; Jong, M. R.; Metselaar, G. A.; Verboom, W.; Huskens, J.; Hulst, R.; Kooijman, H.; Spek, A. L.; Reinhould, D. N. Chem. Eur. J. 2001, 7, 3603.

[128] Zhou, Y. H.; Zhao, M.; Mao, Z. W.; Ji, L. N. Chem. Eur. J. 2008, 14, 7193 .

[129] Tang, S. P.; Chen, S.; Wu, G. F.; Chen, H. Y.; Mao, Z. W.; Ji, L. N. Inorg. Chem. Commun. 2011, 14, 184.

[130] Ikeda, H.; Nishikawa, S.; Yamamoto, Y.; Ueno, A. J. Mol. Catal. A: Chem. 2010, 328, 1.

[131] Six, N.; Menuel, S.; Bricout, H.; Hapiot, F.; Monflier, E. Adv. Synth. Catal. 2010, 352, 1467.

[132] Zhang, H.-C.; Hao, A.-Y.; Li, G.-Z.; Sun, H.-Y. Chin. J. Org. Chem. 2009, 29, 166 (in Chinese). (张华承, 郝爱友, 李干左, 孙宏元, 有机化学, 2009, 29, 166.)

[133] Zhang, H.; Xin, F.; Li, Y.; Hao, A.; An, W.; Sun, T. Prog. Chem. 2010, 22, 2276 (in Chinese).

(张华承, 辛飞飞, 李月明, 郝爱友, 安伟, 孙涛, 化学进展, 2010, 22, 2276.)

[134] Leclercq, L.; Lacour, M.; Sanon, S. H.; Schmitzer, A. R. Chem. Eur. J. 2009, 15, 6327.

[135] Harada, A.; Osaki, M.; Takashima, Y.; Yamaguchi, H. Acc. Chem. Res. 2008, 41, 1143.

[136] Chen, Q.-R.; Liu, C.; Liu, F.-Q. Prog. Chem. 2010, 22, 927 (in Chinese).

(陈清瑞, 刘畅, 刘风岐, 化学进展, 2010, 22, 927.)

[137] Prasannan, A.; Truong, T. L. B.; Hong, P. D.; Somanathan, N.; Shown, I.; Imae, T. Langmuir, 2011, 27, 766.

[138] Zhang, H.; Sun, L.; Liu, Z.; An, W.; Hao, A.; Xin, F.; Shen, J. Colloid Surf., A 2010, 358, 115.

[139] Hu, X.; Lu, Y.; Liu, J. Macromol. Rapid Commun. 2004, 25, 1117.

[140] Feng, J.; Yan, W.; Zhu, J. Synth. Met. 2010, 160, 939.

[141] Badi, N.; Guegan, P.; Legrand, F. X.; Leclercq, L.; Tilloy, S.; Monflier, E. J. Mol. Catal. A: Chem. 2010, 318, 8.

[142] Fang, L; Olson, M. A.; Benitez, D.; Tkatchouk, E.; Goddard, W. A.; Stoddart, J. F. Chem. Soc. Rev. 2010, 39, 17.

[143] Smaldone, R. A.; Forgan, R. S.; Furukawa, H.; Gassensmith, J. J.; Slawin, A. M. Z.; Yaghi, O. M.; Stoddart, J. F. Angew. Chem., Int. Ed. 2010, 49, 8630.

[144] Kang, Y.; Zhou, L.; Li, X.; Yuan, J. J. Mater. Chem. 2011, 21, 3704.

[145] Hamasaka, G.; Muto, T.; Uozumi, Y. Angew. Chem., Int. Ed. 2011, $50,4876$.

[146] Stoll, R. S.; Hecht, S. Angew. Chem., Int. Ed. 2010, 49, 5054.

(Qin, X.) 